\title{
How many patents does it take to signal innovation quality? *
}

\author{
Stefano Comino ${ }^{\dagger} \quad$ Clara Graziano ${ }^{\ddagger}$
}

March 26, 2015

\begin{abstract}
In this paper, we offer a novel explanation to the surge in patenting observed during the last few years. When PTOs (Patent and Trademark Offices) award bad patents, not only do "false innovators" have the incentive to file applications but also, and more interestingly, "true innovators" are forced to patent more intensively in an attempt to signal their type. However, if they are liquidity constrained, true innovators may fail to separate and this fact reduces the incentives to exert effort in R\&D. In addition, drawing on the signaling role of patents highlighted by the model, we investigate some of the proposals that have been put forward in order to mitigate the bad patents problem. We provide an intuitive condition under which a tightening of the patentability standards ("raising the bar") reduces the distortions caused by bad patents. Moreover, we show that introducing a two-tiered patent system is unlikely to improve market outcomes.
\end{abstract}

J.E.L. codes: L13, O31, O34.

Keywords: patenting, bad patents, R\&D incentives, signaling.

*An earlier version of this paper was presented at the EARIE conference (Rome 2012), at the Jornadas de Economia Industrial (Segovia 2013) and at the VI NERI Annual Meeting (Pavia 2014). We are grateful to Reiko Aoki, Giovanni Fonseca, Alberto Galasso, Gerard Llobet, Luigi Pace and David Perez-Castrillo for their comments on the earlier version of the paper.

${ }^{\dagger}$ Corresponding author. Department of Economics and Statistics, University of Udine, Via Tomadini 30/A, 33100 Udine (Italy). E-mail stefano.comino@uniud.it, tel. +390432249211 , fax +390432249229 .

${ }_{\ddagger}$ Department of Economics and Statistics, University of Udine, and CESifo. Address: Department of Economics and Statistics, Via Tomadini 30/A, 33100 Udine (Italy). E-mail clara.graziano@uniud.it, tel. +390432249216, fax +390432249229. 


\section{Introduction}

During the last few years, a dramatic increase in patenting has been accompanied by a rise in the number of so-called "bad patents". As a matter of fact, PTOs (Patent and Trademark Offices) are increasingly granting patent protection to innovations that do not meet the novelty and/or the non-obviousness requirement, and that would not get through a careful examination of the patentability standards. ${ }^{1}$

Since the seminal paper by Farrell and Shapiro (2008), the literature has investigated the economic consequences of bad patents. ${ }^{2}$ According to several commentators, the vast majority of bad patents covers useless technologies or products that no one will ever use and, as such, is economically irrelevant. ${ }^{3}$ As a consequence, PTO examiners should not pay more attention to every application being filed but they should rather concentrate on the few patents that may represent a too heavy burden to future innovators. This argument is clearly summarized by the following quote taken from Lemley et al. (2005, page 12): "The problem, then, is not that the Patent Office issues a large number of bad patents. Rather, it is that the Patent Office issues a small but worrisome number of economically significant bad patents...". However, this view overlooks an important role that patents play. When some significant characteristic of the inventor is not observable, then patents might serve as a quality signal for third parties, such as potential investors or competitors (see Long, 2002 and Schankerman, 2013).

Several empirical studies demonstrate the growing importance of patents as signaling devices, especially for start-ups and, more generally, for SMEs. These companies have little or no track record, and therefore face more serious informational problems. For instance, Hsu and Ziedonis (2008) look at US semiconductor firms that received venture financing and show that

\footnotetext{
${ }^{1}$ The issue is particulary serious for the U.S. Patent and Trademark Office. Lemley and Sampat (2008) report that between $75 \%$ and $97 \%$ of patent applications filed in the U.S. is finally approved and, rather provocatively, they ask themselves whether the USPTO has become a rubber-stamp that grants patents to every application being filed.

${ }^{2}$ The role of licensing negotiations in mitigating the consequences of bad patents is investigated in Farrell and Shapiro (2008) and in Choi (2005). Caillaud and Duchene (2011), on the other hand, focus on the overload problem at the PTO and demonstrate the possible emergence of a "low R\&D equilibrium". In such an equilibrium, firms invest little in R\&D, they file many applications some of which based on bogus ideas, and the (overloaded) PTO grants bad patents with large probability.

${ }^{3}$ Lemley et al. (2005) report a series of curious patents awarded by the USPTO, such as patents "covering obvious inventions like a crustless peanut butter and jelly sandwich, ridiculous ideas like a method of exercising a cat with a laser pointer, and impossible concepts like travelling faster than the speed of light."
} 
having a large stock of patent applications increases both the likelihood of the company being financed by venture capitalists as well as the amount of financial aid received. By using their estimates, Gambardella (2013) calculates that the value of patents as quality signals could be as high as US $\$ 1.2$ million, though he suggests that 93 thousand is a more reasonable estimate. Similarly, the recent Berkeley Patent Survey shows that the top-ranked motivations to patent for technological start-ups are the increased chances of securing outside investment and the enhancement of the company's reputation, both suggesting that patenting occurs for signalling purposes (see Graham et al., 2010). Additional empirical evidence in favor of the signaling role of patents is provided by Mann (2005), Cockburn and MacGarview (2009), Häussler et al. (2009), Conti et al. (2013a), and by Greenberg (2013). ${ }^{4}$

Clearly, when the PTO issues a significant number of bad patents, the credibility of the information conveyed through the patenting process can be seriously undermined. When "true innovators" as well as "false innovators" get through the examination process at the PTO, patents become a noisy signal about the quality of the inventor/innovation. ${ }^{5}$

A couple of recent theoretical papers investigate the signaling role of patents when the screening of the PTO is of poor quality. Koenen and Peitz (2013) model an infinite horizon game in which, at each period of time, the firm generates a patentable idea. The two authors determine the conditions under which reputational concerns induce the firm to only apply for a patent when it has generated a true innovation (and therefore refrain from filing bad applications based on bogus ideas). Atal and Bar (2014) focus on one of the proposals for mitigating the bad patents problem suggested in the literature, namely the introduction of a two-tiered patent system where inventors are free to apply for a "gold-plated" patent (with higher fees and tighter PTO scrutiny but also offering stronger protection for the invention) or for a "regular" patent. Although, the authors show that introducing a second patent-tier reduces the incidence of bad patents, they also prove

\footnotetext{
${ }^{4}$ Conti et al. (2013b) on the other hand look at two signals firms may use (patents and capital invested by the entrepreneur in the venture) and show that venture capitalists care more about patents, while business angels are more concerned with the money the founder has invested.

${ }^{5}$ The examination process at PTOs can be very long and one may wonder whether their decisions actually convey valuable information to third parties. As a matter of fact, PTOs reveal important information on applications before taking the final approval/rejection decision. For instance, within 18 months of the application being filed, the European Patent Office (EPO) publishes the search report where the references that call the novelty or the inventive step of a claim into question (the so-called $\mathrm{X}$ and $\mathrm{Y}$ references) are listed; applications with many $\mathrm{X}$ and $\mathrm{Y}$ references are likely to be ultimately rejected. Greater details on the examination process at the EPO can be found in Harhoff and Wagner (2009).
} 
that economically more significant innovations do not necessarily end up in applications for gold-plated patents.

In this paper, we focus on a different mechanism true innovators might use in order to signal their type, namely the number of applications they file. ${ }^{6}$ As a matter of fact, firms are often involved in various $R \& D$ projects and, therefore, they may file several patent applications. Moreover, there is no one-to-one correspondence between innovations and patents and a single new product or process may be covered by a series of patents, some of them applied possibly for some ancillary/secondary aspects of the innovation. ${ }^{7}$

In the following sections, we consider a start-up company with limited financial resources involved in a multi-stage innovation game in which patents just have a signaling role (they are used to signal whether the firm is a "true" or a "false" innovator). In the first stage, the firm observes the financial resources needed to complete the research project and chooses whether to make the investment. Should it choose to invest, the innovation is developed and the firm becomes a "true innovator"; if it does not, the innovation does not materialize and we say that the firm becomes a "false innovator". In the second stage, the firm decides how many patent applications to file. However, since the PTO does not screen applications perfectly, the false innovator also has an incentive to file applications. The maximum number of patents the firm can apply for is determined by the financial resources remaining after the investment decision. Hence, the assumption of limited financial resources implies that, even though a true innovator benefits most from signaling, the false innovator is endowed with a greater budget for patenting, as it did not invest during the previous stage. We show that as a result of the imperfections in the PTO examination process, true innovators increase the number of applications filed in an attempt to signal their type. However, if they are liquidity constrained at the patenting stage they are unable to separate from the false innovator; hence, they collect lower revenues. In turn, as we show in the Extensions section, the inability of the true innovators to signal their type reduces $R \& D$ incentives.

Our paper contributes to the recent literature addressing the growing concerns for the increasing numbers of patents that allegedly fail to meet

\footnotetext{
${ }^{6}$ The number of applications as a signal of quality is also considered by Conti et al. (2013a). However, they do not consider the issue of bad patents, and they assume that when deciding how many patents to apply for the innovator is never liquidity constrained, which is one of the key drivers of our analysis.

${ }^{7}$ In a study on the pharmaceutical industry, Ouellette (2010) reports that, on average, 3.5 patents cover one single drug increasing to about 5 in the case of blockbuster drugs. For the use of patents covering ancillary aspects of the innovation, see Hemphill and Sampat (2012).
} 
the novelty or non-obviousness requirement and offers a new explanation to the recent surge in patenting. While the literature suggests that, especially in high-tech sectors, companies amass large patent portfolios in order to use them strategically during negotiations or even to preempt competitors, ${ }^{8}$ we argue that the observed rise in patenting can also be explained by poor PTO screening coupled with the signaling role of patents. ${ }^{9}$ In our model, the rise in patenting is not simply due to the rather obvious fact that the false innovator takes advantage of low PTO standards to file numerous applications. More interestingly, the increase may result from the fact that true innovators are induced to rise the number of applications in an attempt to signal their type.

As argued above, the signaling motive for patenting seems to be particularly important for start-ups and SMEs. Similarly, the assumption of a limited budget available for patenting and research, is more suited to characterize the behaviour of start-up companies and small enterprises. For instance, the existence of a trade-off between resources spent on patenting and investment in R\&D activities was shown by Mann (2005) in a study on the software industry. The author reported an interview with a software developer who explicitly acknowledged that "Every dollar we spend on [patenting] is a dollar we can't spend on a software engineer (pp. 982-3)". A trade-off between patenting and R\&D is also suggested by the size of spending on the patent application process. According to Graham et al. (2010) the estimated cost of obtaining a patent in the U.S., including attorney fees, is $\$ 35,000$. As regards the E.U., in a study prepared for the European Patent Office, Roland Berger (2005) estimates that the overall cost of filing an application is between 30,000 and 46,000 euros. These figures clearly show that the cost of patenting may be substantial, especially for start-ups or SMEs. Indeed, the technological start-ups included in the Berkeley Patent Survey reported that the main reason they choose not to apply for patent protection was the cost of the application process. The second most important reason was the cost of enforcing patents (see Graham et al., 2010). Similarly, in a study on patenting behavior in the UK, Hall et al. (2013) observed that the low propensity to patent of SMEs is most likely explained by the high costs related to both the application process as well as the monitoring, enforcement and, possibly, litigation of the IP rights.

A series of recent theoretical papers focuses on the informational content revealed by the decision of whether to patent or keep the innovation secret

\footnotetext{
${ }^{8}$ See Hall and Ziedonis (2001) among others.

${ }^{9}$ This fact is also suggested implicitly in Long (2002). When patents stocks convey information, then there are incentives "to patent the smallest publishable unit, and divide what would normally be a single patent on an invention into multiple smaller patents on different facets of the same invention."
} 
and/or by the decision of how much innovation-related information to disclose (Anton and Yao, 2003 and 2004, Jansen, 2011 and Fabrizi et al, 2013). This literature does not focus on the bad patents issue which, instead, is our main concern. ${ }^{10}$ More concretely, our paper differs from these contributions in two fundamental ways. Firstly, the PTO is not explicitly modelled in this literature: all patent applications are accepted and, consequently, signaling only occurs through the strategies chosen by the firm. In Anton and Yao (2003) and Jansen (2011) patents can be invalidated ex-post by the Court; but, such a decision does not convey any information about the firm's type. By contrast, in our paper, we assume that information is revealed both by the strategy chosen by the firm as well as by the PTO's decisions. The Patent and Trademark Office is more likely to accept applications filed by the true innovator and, hence, its acceptance/rejection decisions reveal useful information about the firm's type. The importance of the PTO's decisions for signaling purposes is supported by the above evidence according to which granted patents increase the chances of being backed by VCs. The second major difference of our model rests on the assumption of limited financial resources that might constrain the choices of the firm. These two assumptions enable us to develop a model in which bad patents emerge as an equilibrium phenomenon and true innovators patent intensively in an attempt to signal their type.

Interestingly, our paper is consistent with a puzzling result that emerges in the empirical literature looking at the signaling role of patents. While the stock of patent applications appears to significantly improve the chances of being financed, findings regarding granted patents are contrasting: in some studies they do not add anything (e.g. Haüssler et al, 2009) while in others they further increase the likelihood of receiving financial aid (e.g. Greenberg, 2013). This mixed evidence cannot be reconciled with theoretical contributions mentioned above. However, it is consistent with the main result of our model in which in some cases the true innovator is able to separate through the number of applications it files, while in others it is prevented from separating by the financial constraint. As a result, the decision of the PTO is redundant in the former cases but it is informative in the latter ones.

In the paper, we also discuss some of the proposals that have been put forward in order to mitigate the bad patents problem. We provide an intuitive condition under which an improvement in the screening ability of the PTO, coupled with an increase in the patenting fees ("raising the bar"), is likely

\footnotetext{
${ }^{10}$ In Anton and Yao (2003) and (2004) and in Jansen (2011) only true innovators were considered. Fabrizi et al. (2013) analysed the case of a firm possessing either a "good idea" or a "bad idea", with the latter requiring greater costs in order to be patented. However, the authors show that in equilibrium only good ideas are patented.
} 
to reduce the distortions caused by the presence of bad patents. Moreover, we show that a two-tiered patent system is likely to be ineffective once we depart from the standard assumption that the firm chooses either zero or one patent. When the firm also chooses the number of patents to apply for, it is able to endogenously "gold-plate" its innovation. Hence, introducing a two-tiered patent system is unlikely to increase market efficiency.

The paper is organized as follows. The model is introduced in Section 2, while in Section 3 we derive the equilibrium of the game and the main results. The policy implications of our findings are discussed in Section 4, and in Section 5 there are some extensions and a discussion of the main assumptions of the model. Finally, Section 6 concludes. All proofs can be found in the Appendix which also contains some microfoundations of the reduced form of payoff we consider in the paper.

\section{The Model}

A start-up company endowed with an idea for an R\&D project and with financial resources $K$, is involved in the following three-stage innovation game. In the first stage, the firm observes the investment $i$ necessary to complete the R\&D project. Should the investment be undertaken, the innovation is developed and the firm "becomes a true innovator"; if the investment is not undertaken, the innovation does not materialize and the firm "becomes a false innovator". During the second stage of the game, the firm chooses how many patent applications, $n \geq 0$, to file at the Patent and Trademark Office. As we clarify below, the PTO's screening of the patentability requirements is not perfect and this may also encourage the false innovator to file patent applications. In the last stage of the game, payoffs are realized. More specifically, the three stages of the game are as follows.

$t=1$ : investment stage

The firm privately observes the amount of investment required to complete the project: $i \in\left\{I_{1}, I_{2}, I_{3}\right\}$, with $I_{1}<I_{2}<K<I_{3}$. Clearly, when $i=I_{3}$ is observed, the firm does not have enough financial resources to proceed. Hence it terminates the project becoming a false innovator. By contrast, we assume that $I_{1}$ and $I_{2}$ are small enough and such that the R\&D project is not only viable but also profitable. Hence when $i=I_{1}$ or $i=I_{2}$ the investment is undertaken. We assume that the innovation technology is deterministic: whenever the investment is undertaken the innovation realizes and we say that the firm becomes a true innovator. The probabilities of the different realizations of $i$ are $p_{1}, p_{2}$ and $p_{3}$, respectively, with $p_{1}+p_{2}+p_{3}=1$; these 
probabilities are publicly known.

t=2: patenting stage

In the second stage, the firm chooses the number of applications, $n \geq 0$, to file at the Patent and Trademark Office. For the sake of simplicity, in what follows we consider $n$ as a continuous variable. ${ }^{11}$ Moreover, we assume that patents are only a device to signal the firm's own type (true or false innovator) to the market, and we abstract from any other motivation, such as protective reasons, that may induce the firm to patent.

The PTO does not have the time or resources to conduct an accurate screening of applications, and its examination of the patentability requirements is not perfect. As a result of this, the PTO may grant patents also to the false innovator.

We assume that the firm is free to file any number of applications, with the only constraint represented by the financial resources remaining after the investment stage. Formally, if we let $P$ denote the patenting fees, the firm can apply at most for $n \leq(K-I) / P \equiv \bar{n}(I)$, where $I$ is the amount invested at $t=1$, with $I=i$ if the firm has made the investment and $I=0$ in the case it did not invest.

We assume that each application by the true innovator is accepted by the PTO, and, by contrast, the applications by the false type may be either accepted or rejected. Hence, the number of patents granted by the PTO equals $n$ if the applicant is a true innovator while it may be smaller for the false innovator. Clearly, when the PTO grants less than $n$ patents, its decision is fully informative: it "certifies" that the firm is a false innovator. On the contrary, when $n$ patents are granted, then the PTO's decision is not fully informative and the applicant may either be a true or a false innovator.

Formally, we let $g(\theta, n) \in[0,1]$ denote the probability that the PTO's decision is fully informative; in other words, when the false innovator files $n$ applications, $g(\theta, n)$ is the probability that the PTO grants less than $n$ patents. The parameter $\theta$ measures the accuracy of the PTO's decision.

We assume that $g(\theta, n)$ is differentiable in its two arguments and such that:

Assumption $\left.1: i) \frac{\partial g(\theta, n)}{\partial \theta}>0, i i\right) \frac{\partial g(\theta, n)}{\partial n}>0$ and $\left.i i i\right) \frac{\partial^{2} g(\theta, n)}{\partial n \partial \theta} \geq 0$.

Assumption 1-i) implies that the more accurate the PTO's screening process (the larger $\theta$ ) the higher the probability that some of the false innovator's

\footnotetext{
${ }^{11}$ For expositional convenience, we refer to $n$ as the "number" of patent applications, even though, with a continuous variable, it would be more appropriate to talk about the "mass" of applications.
} 
applications are rejected. Similarly, assumption 1-ii) requires that the likelihood of the PTO's decision being fully informative increases with the number of applications. As $n$ rises, the applicant reveals more information during the patenting process making it easier for the PTO to figure out whether the innovation has been developed. ${ }^{12}$ Finally, assumption 1-iii) implies that there is weak complementarity between the number of applications and the PTO accuracy.

Clearly, when the firm does not file any application $(n=0)$ the PTO takes no decision, and no information is revealed, $g(\theta, 0)=0$.

$t=3$ : payoff stage

We model the last stage of the game in a reduced-form manner ${ }^{13}$ where we assume that the payoff depends on: i) whether the firm is a true or a false innovator, and ii) the market's beliefs about the firm type. The beliefs are based on the number of applications filed by the firm and on the PTO's decision. Consequently, as argued previously, in our model patenting only has a signaling role.

Formally, let $\xi(1-\xi)$ denote the belief associated with the event "the firm is a true innovator" ("the firm is a false innovator" respectively). Clearly, when $n$ applications are filed, $\xi=0$ if the PTO grants fewer than $n$ patents while we let $\xi=\xi_{n}$ if $n$ patents are awarded. More concretely, the payoffs are as follows:

- with probability $f(\xi)$ the firm earns a large profit: the false innovator earns $R$, the true innovator earns $R l$, with $l \geq 1$;

- with probability $1-f(\xi)$ the firm earns a small profit: the false innovator earns $r$, the true innovator earns $r l$, with $0 \leq r<R$.

Therefore, when the market holds the belief $\xi$, the payoff that the false and the true innovator expect at stage $t=3$ is: ${ }^{14}$

$$
\begin{aligned}
f(\xi) R+(1-f(\xi)) r & =f(\xi) \Delta+r, \\
f(\xi) R l+(1-f(\xi)) r l & =f(\xi) \Delta l+r l,
\end{aligned}
$$

respectively and where $\Delta \equiv R-r$.

\footnotetext{
${ }^{12}$ Assumption 1-ii) is in line with the literature on information disclosure at the patenting stage. For instance, Anton and Yao (2004) assume that the broader the patent protection applied for (the larger $n$ in our setting) the more information the firm is required to disclose.

${ }^{13}$ In the Appendix, we provide three possible microfoundations for the reduced-form payoff we employ

${ }^{14}$ Expected payoffs are computed net of $K, i$ and $n P$.
} 
By resorting to one of the microfoundations provided in the Appendix, we can interpret the above payoff structure in the following manner. At $t=3$ the firm needs to be financed by a venture capitalist in order to bring the innovation to market. The probability of being financed is $f(\xi)$, and depends on the beliefs that the venture capitalist holds. The payoff the firm collects depends on its type and on the probability of being financed.

Throughout the paper we assume that $f(\xi)$ is differentiable and increasing in $\xi$. Using the previous interpretation of the model, this means that the venture capitalist is more likely to finance the firm as the probability that it attaches to the event "the firm is a true innovator" increases. Notice that for any beliefs, the payoff of the true innovator is $l$ times larger than that of the false type. This assumption, combined with the behavior of the PTO, implies that we are postulating a positive correlation between the chances of being granted patents and the payoffs that the two types of the firm are able to collect. ${ }^{15}$

We look for the Perfect Bayesian Equilibrium (PBE) of the game and we require the out-of-equilibrium beliefs to be "reasonable"; in particular, we require them to satisfy the D1 criterion. ${ }^{16}$

\section{Equilibrium at the patenting stage}

At the patenting stage, two things matter: i) whether the firm has developed the innovation (i.e. whether it is a true or a false innovator) and ii) the amount of financial resources that it can use to file patent applications. From the investment stage, it follows that the firm can actually be of three different types. $^{17}$ If it did not complete the project it is a false innovator that can apply for at most $\bar{n}(0)=K / P$ patents. If instead it developed the innovation it can be of two different types according to the amount of resources invested at $t=1$. The firm can be a true innovator that has spent $I_{2}$ and that

\footnotetext{
${ }^{15}$ We believe it is reasonable to assume that the true innovator who has completed the project resulting in an innovation obtains a higher profit than the false type. Moreover, our assumption resembles the positive correlation between innovativeness and profitability commonly postulated in the innovation literature (see O'Donoghue, 1998 and Fabrizi et al., 2013). Some authors make an even stronger assumption by representing innovativeness and profitability with the same variable (see O'Donoghue, Scotchmer and Thisse, 1998).

${ }^{16}$ See Banks and Sobel (1987).

${ }^{17}$ The case we focus on (two true innovators and a false type) is the most interesting. If the investment $I_{2}$ is not profitable, there are two firm types at the patenting stage: a false innovator (which has observed either $I_{2}$ or $I_{3}$ ) and a deep-pocket true innovator. It would be easy to prove that under Assumption 2 the equilibrium of the patenting subgame would be separating: the false innovator would not apply for any patent while the true innovator would apply for the minimum number of patents the false innovator is not willing to file.
} 
therefore can apply for at most $\bar{n}\left(I_{2}\right)=\left(K-I_{2}\right) / P$ patents; for reasons that we clarify below, we refer to this type of the firm as the "liquidity-constrained true innovator". Alternatively, it can be a true innovator that has spent $I_{1}$ and that, as a result, can file at most $\bar{n}\left(I_{1}\right)=\left(K-I_{1}\right) / P$ applications; we refer to this type of the firm as the "deep-pocket true innovator". For the sake of simplicity, when we refer to both the liquidity-constrained and the deep-pocket true innovators we just say "true types".

The next lemma proves a very important preliminary result concerning the patenting game. Suppose, for the time being, that the constraint $n \leq$ $\bar{n}(I)$, with $I=0, I_{1}, I_{2}$, does not apply, and that the firm is able to file whatever number of patents $n$ it likes. According to Lemma 1, the true types are the ones that have more incentives to increase the number of applications.

Lemma 1 (Benchmark: no constraint on $n$ ) Suppose $n$ is chosen with some positive probability by the false innovator and by at least one of the true types; then in any PBE satisfying the D1 criterion, the true types prefer $n+\varepsilon$ to $n$, where $\varepsilon$ is a positive, negligible number.

If there were no financial constraints to the number of applications the firm can file, then the true types certainly would prefer to increase $n$ in order to improve market beliefs, and possibly separate from the false innovator. The reason why this occurs is twofold. The false innovator is less willing to increase the number of applications: as $n$ rises, the probability of the PTO certifying the firm to be the false innovator also increases. Moreover, as shown in (1), the true types benefit more than the false innovator from an improvement in market beliefs: an increase in $\xi$ implies a benefit proportional to $\Delta l$ for the true types, and proportional to $\Delta$ for the false innovator, with $\Delta l \geq \Delta$.

Let us now consider the behavior of the firm when the financial constraints $n \leq \bar{n}(I)$, with $I=0, I_{1}, I_{2}$ are in place. In particular, in what follows, we focus on the most interesting case in which $\bar{n}\left(I_{1}\right)$ is so large that the deeppocket true innovator can separate, while $\bar{n}\left(I_{2}\right)$ is sufficiently low to prevent the liquidity-constrained true innovator from separating. ${ }^{18}$

Assumption 2 i) $\left.\bar{n}\left(I_{1}\right) P>\left(1-g\left(\theta, \bar{n}\left(I_{1}\right)\right)\right)(f(1)-f(0)) \Delta\right)$ and ii) $\bar{n}\left(I_{2}\right) P<$ $\left.\left(1-g\left(\theta, \bar{n}\left(I_{2}\right)\right)\right)(f(1)-f(0)) \Delta\right)$.

\footnotetext{
${ }^{18}$ It can be proved that if assumption 2-ii) is not satisfied, in the unique equilibrium the true types separate from the false innovator by applying a sufficiently large number of patents. By contrast, if condition 2-i) is not satisfied then in the unique equilibrium the true types apply for the maximum number of patents they can afford, $\bar{n}\left(I_{2}\right)$ and $\bar{n}\left(I_{1}\right)$, while the false innovator randomizes between $\bar{n}\left(I_{2}\right)$ and $\bar{n}\left(I_{1}\right)$ (and possibly $n=0$ ).
} 
Assumption 2-i) ensures that the deep-pocket true innovator is able to separate from the false type. More specifically, the assumption implies that the false innovator does not benefit from mimicking the deep-pocket true innovator that applies for the maximum affordable number of patents. Formally, the condition ensures that the false innovator prefers not to file any application $(n=0)$, revealing its type, rather than imitating the deep-pocket true innovator, and filing $\bar{n}\left(I_{1}\right)$ patents. In the former case, the false type obtains $f(0) \Delta+r+K$. In the latter case, it is detected with probability $g\left(\theta, \bar{n}\left(I_{1}\right)\right)$ and obtains $g\left(\theta, \bar{n}\left(I_{1}\right)\right) f(0) \Delta+\left(1-g\left(\theta, \bar{n}\left(I_{1}\right)\right)\right) f(1) \Delta+r+K-$ $\bar{n}\left(I_{1}\right) P$.

Putting together Lemma 1 (the deep-pocket true innovator prefers to separate from the false type) and Assumption 2-i) (separation is financially viable) it follows that:

Lemma 2 In any PBE satisfying the D1 criterion, the deep-pocket true innovator separates from the false type.

By contrast, Assumption 2-ii) implies that $\bar{n}\left(I_{2}\right)$ is low enough for the false type to benefit from imitating the liquidity-constrained true innovator, even if $\bar{n}\left(I_{2}\right)$ applications are filed. Formally, Assumption 2-ii) implies that the false type prefers $\bar{n}\left(I_{2}\right)$ (with an associated payoff of $g\left(\theta, \bar{n}\left(I_{2}\right)\right) f(0) \Delta+$ $\left.\left(1-g\left(\theta, \bar{n}\left(I_{2}\right)\right)\right) f(1) \Delta+r+K-\bar{n}\left(I_{2}\right) P\right)$ to $n=0$ (with an associated payoff of $f(0) \Delta+r+K)$. An implication of Assumption 2-ii) is the following:

Lemma 3 There is no PBE in which the false innovator separates with probability 1.

By combining Assumption 2-ii) (the false innovator benefits from imitating the liquidity-constrained true innovator) and Lemma 1 (the liquidityconstrained true innovator benefits from choosing $n+\varepsilon$ when $n$ is chosen by the false innovator) we are able to derive the optimal strategy chosen by the liquidity-constrained true innovator in any equilibrium of the patenting subgame.

Lemma 4 In any PBE satisfying the D1 criterion, the liquidity-constrained true innovator applies for the maximum affordable number of patents, $\bar{n}\left(I_{2}\right)$, with probability 1.

We are now in the position to determine the unique equilibrium of the patenting stage that satisfies the D1 criterion. 
Proposition 1 The unique PBE satisfying the D1 criterion is the following:

- the false innovator applies for $\bar{n}\left(I_{2}\right)$ patents with probability $h\left(\bar{n}\left(I_{2}\right)\right) \in$ $(0,1]$ and chooses $n=0$ with complementary probability;

- the liquidity-constrained true innovator applies for $\bar{n}\left(I_{2}\right)$ patents;

- the deep-pocket true innovator applies for $n^{T}>\bar{n}\left(I_{2}\right)$ patents $\left(n^{T}\right.$ is defined in the Appendix).

The beliefs are:

- when the PTO certifies the firm to be the false innovator, $\xi=0$;

- when the PTO does not certify the firm to be the false innovator: if $n \geq n^{T}$, then $\xi_{n}=1$; if $n=\bar{n}\left(I_{2}\right)$, then $\xi_{\bar{n}\left(I_{2}\right)}=p_{2} /\left[p_{2}+p_{3}(1-\right.$ $\left.\left.g\left(\theta, \bar{n}\left(I_{2}\right)\right)\right) h\left(\bar{n}\left(I_{2}\right)\right)\right] ; \xi_{n}=0$ in all other cases.

As already shown in Lemma 4, the liquidity-constrained true innovator applies for the maximum affordable number of patents, $\bar{n}\left(I_{2}\right)$, and the false type imitates it with a positive probability denoted by $h\left(\bar{n}\left(I_{2}\right)\right)$. Hence, despite applying for the largest number of patents it can file, the liquidityconstrained true innovator is unable to separate from the false type. In the Appendix, we show that when $\bar{n}\left(I_{2}\right) P$ is low, then $h\left(\bar{n}\left(I_{2}\right)\right)=1$; in other words, when the cost of imitating the liquidity-constrained true innovator is low, the false type mimics it with probability 1 . For expositional convenience in the following we refer to this situation as the low-imitation-cost case. For larger values of $\bar{n}\left(I_{2}\right) P$, the false type imitates the liquidity-constrained true innovator with a probability $h\left(\bar{n}\left(I_{2}\right)\right)$ strictly lower than 1 . In other words, when the cost of imitating the liquidity-constrained true innovator gets larger, the false type plays mixed strategies. We denote this situation as the highimitation-cost case.

When $\bar{n}\left(I_{2}\right)$ is observed, market beliefs depend crucially on the PTO decision. If the Patent Office rejects at least one application, the firm is known to be the false innovator and $\xi=0$; if all applications are accepted, the market updates its belief according to Bayes' rule and equilibrium strategies and $\xi_{\bar{n}\left(I_{2}\right)}=p_{2} /\left\{p_{2}+p_{3}\left[\left(1-g\left(\theta, \bar{n}\left(I_{2}\right)\right)\right) h\left(\bar{n}\left(I_{2}\right)\right)\right]\right\}$.

In contrast to the liquidity-constrained, the deep-pocket true innovator is able to separate by filing a sufficiently large number of patents $n^{T}\left(>\bar{n}\left(I_{2}\right)\right)$, thus making it too costly for the false type to imitate this strategy. In this case, the decision taken by the PTO is irrelevant since the market, by simply observing $n^{T}$, can infer the type of innovator and $\xi_{n}=1$. As shown in the Appendix, $n^{T}$ is the number of applications that makes the false innovator indifferent between choosing $n^{T}$ and playing the equilibrium strategy, $\bar{n}\left(I_{2}\right)$.

In summary, Proposition 1 highlights some interesting consequences of the imperfect screening by the PTO. Firstly, the false innovator gets the 
chance to disguise its type by filing applications. Secondly, the true types increase the number of patents they file in an attempt to credibly signal that they have developed the innovation; the liquidity-constrained true innovator devotes its entire budget to filing applications while the deep-pocket true innovator raises $n$ up to the point where the false innovator does not profit from imitating its behavior. Finally, in equilibrium, the PTO decision determines the market beliefs but only in the case of $\bar{n}\left(I_{2}\right)$ i.e. only when the firm is unable to separate through the number of applications. By contrast, when $n$ is a separating strategy, the PTO decision is irrelevant.

\section{Comparative Statics}

The above analysis has shown that "overpatenting" is the optimal reaction to the low patentability standards applied by the PTO. This finding is reinforced by the comparative statics analysis we carry out in this section.

We start by determining the effect of an increase in $\theta$ on the number of patents filed by true types.

Corollary 1 In equilibrium the overall number of patents applied for by true types decreases as $\theta$ increases.

The number of applications filed by the liquidity-constrained true innovator does not depend on $\theta$ (recall that $\bar{n}\left(I_{2}\right)=\left(K-I_{2}\right) / P$ ). Hence, the reduction in applications by true types following an increase in $\theta$ is driven by the behavior of the deep-pocket true innovator. This type of firm applies for $n^{T}$ patents defined as the number of applications that makes the false innovator indifferent between the equilibrium strategy, $\bar{n}\left(I_{2}\right)$, and $n^{T}$ itself. As shown in the Appendix, as $\theta$ increases, $\bar{n}\left(I_{2}\right)$ becomes comparatively more profitable for the false innovator than $n^{T}$. Hence, to restore indifference between these two strategies $n^{T}$ has to diminish. Indeed, an increase in $\theta$ makes it more likely for the PTO to detect the false innovator, thus reducing its expected payoff; since $\partial^{2} g(\theta, n) / \partial n \partial \theta \geq 0$, this (negative) effect is stronger when $n^{T}$ rather than $\bar{n}\left(I_{2}\right)$ is chosen (recall that $n^{T}>\bar{n}\left(I_{2}\right)$ ). Moreover, when $\bar{n}\left(I_{2}\right)$ is chosen and the false innovator is not detected, $\xi_{\bar{n}\left(I_{2}\right)}$ increases, thus augmenting the payoff for this firm type.

The effect of an increase in $\theta$ on the number of applications filed by the false innovator depends on whether the cost of imitating the liquidityconstrained true innovator is small or large. In the low-imitation-cost case, the false innovator applies for $\bar{n}\left(I_{2}\right)=\left(K-I_{2}\right) / P$ patents, a choice which is not affected by $\theta$. Therefore, Corollary 1 implies that the overall number of applications also decreases as $\theta$ gets larger. 
On the contrary, in the high-imitation-cost case, $\theta$ impacts on the mixed strategies played by the false innovator due to two opposing effects. On the one hand, the probability $g(\theta, n)$ that the false innovator is detected increases, thus reducing its payoff from $f\left(\xi_{\bar{n}\left(I_{2}\right)}\right) \Delta+r$ to $f(0) \Delta+r$; this, in turn, decreases $h\left(\bar{n}\left(I_{2}\right)\right)$, the probability that $\bar{n}\left(I_{2}\right)$ is selected. On the other hand, with probability $\left(1-g\left(\theta, \bar{n}\left(I_{2}\right)\right)\right)$ the false innovator is not detected and the consequent improvement in beliefs leads to a payoff rise equal to $\frac{d f(\xi)}{d \xi_{\bar{n}\left(I_{2}\right)}} \frac{\partial \xi_{\bar{n}\left(I_{2}\right)}}{\partial g\left(\theta, \bar{n}\left(I_{2}\right)\right)} \frac{\partial g\left(\theta, \bar{n}\left(I_{2}\right)\right)}{\partial \theta} \Delta$; this second effect increases $h\left(\bar{n}\left(I_{2}\right)\right)$. Therefore, when condition

$$
f\left(\xi_{\bar{n}\left(I_{2}\right)}\right)-f(0) \geq\left(1-g\left(\theta, \bar{n}\left(I_{2}\right)\right)\right) \frac{d f(\xi)}{d \xi_{\bar{n}\left(I_{2}\right)}} \frac{\partial \xi_{\bar{n}\left(I_{2}\right)}}{\partial g\left(\theta, \bar{n}\left(I_{2}\right)\right)} \frac{\partial g\left(\theta, \bar{n}\left(I_{2}\right)\right)}{\partial \theta}
$$

is satisfied, the former (negative) effect dominates, and a rise in $\theta$ reduces the expected number of false type applications $\left(h\left(\bar{n}\left(I_{2}\right)\right) \bar{n}\left(I_{2}\right)\right)$. Therefore, we can conclude that:

Corollary 2 In the low-imitation-cost case, an increase in $\theta$ always leads to a decrease in the overall number of applications. In the high-imitationcost case, condition (2) is sufficient to guarantee that the overall number of applications decreases as $\theta$ gets larger.

\section{$4 \quad$ Policy proposals}

Various proposals for fixing the bad patents problem and reducing the number of applications based on bogus ideas have been put forward by economists, lawyers and practitioners. Below, we use our model to discuss the possible effects of some of these proposals.

\subsection{Rasing the bar}

As advanced by several authors, a possible fix to the bad patents problem is to "raise the bar", that is, to make the PTO screening more stringent (see for example Encaoua et al. 2006). In terms of our model, "raising the bar" can be interpreted as an increase in $\theta$, the accuracy of the screening activity. ${ }^{19}$ In

\footnotetext{
${ }^{19}$ Kou et al. (2013) and Schuett (2013) also look at the effect of a tightening of patentability standards. One main difference from our model is that in the setting proposed patents are not used for signaling purposes (innovators always apply for one patent). Their focus is on how, a strengthening of the PTO examination process affects the incentive to perform research activities and/or to develop the innovation.
} 
Corollaries 1 and 2 we have already seen how a rise in $\theta$ affects the number of patent applications. However, the previous findings were derived under the assumption that the patenting fees $P$ are constant. Clearly, when the PTO is self-funded, even partially, a rise in $\theta$, by requiring to devote more resources to screening patent applications, might result in raising patenting fees in order to offset the increase in costs. To capture this effect, we now assume that the patenting fees are a (weakly) increasing function of $\theta: d P / d \theta \geq 0$. Hence, the following analysis is a generalization of the results shown in Corollaries 1 and 2.

The effect of raising the bar crucially depends on how a larger $\theta$ affects $g\left(\theta, \bar{n}\left(I_{2}\right)\right)$. As a matter of fact, in equilibrium, the PTO's decisions only affect the beliefs that the market holds when $\bar{n}\left(I_{2}\right)$ is observed. In the following, we refer to $g\left(\theta, \bar{n}\left(I_{2}\right)\right)$ as the "probability of correct screening", i.e. the probability that the PTO correctly certifies that the firm is the false type.

Notice that when $d P / d \theta \geq 0$, an increase in $\theta$ entails two opposing effects on $g\left(\theta, \bar{n}\left(I_{2}\right)\right)$, as highlighted by the expressions here below:

$$
[\underbrace{\frac{\partial g\left(\theta, \bar{n}\left(I_{2}\right)\right)}{\partial \theta}}_{(+)}+\underbrace{\frac{\partial g\left(\theta, \bar{n}\left(I_{2}\right)\right)}{\partial n}}_{(+)} \underbrace{\frac{\partial \bar{n}\left(I_{2}\right)}{\partial P}}_{(-)} \underbrace{\frac{d P}{d \theta}}_{(\geq 0)}] .
$$

The first term/effect is positive since, by Assumption 1, $g(\theta, n)$ increases with $\theta$. On the contrary, the second term/effect is negative: when $d P / d \theta \geq 0$, an increase in $\theta$ reduces $\bar{n}\left(I_{2}\right)$, thus lowering the probability that the PTO certifies the false innovator $(g(\theta, n)$ increases with $n)$.

The effect of a larger $\theta$ on the number of applications filed by the true types is clear-cut:

Proposition 2 Suppose that patenting fees are a weakly increasing function of the PTO's accuracy, $d P / d \theta \geq 0$. In this case, if "probability of correct screening", $g\left(\theta, \bar{n}\left(I_{2}\right)\right)$, increases with $\theta$, then the number of patents that true types apply for decreases as $\theta$ rises.

For similar reasons to Corollary 1 the number of applications filed by the deep-pocket true innovator, $n^{T}$, falls as $\theta$ increases. The effect of tighter screening by the PTO on the number of applications by the liquidity-constrained true innovator is clear; as already observed, when $d P / d \theta \geq 0$, as $\theta$ increases $\bar{n}\left(I_{2}\right)$ decreases in order to satisfy the constraint.

Similarly to the comparative static analysis conducted in the previous section, the effect of a larger $\theta$ on the number of applications filed by the 
false type depends on the cost of imitating the strategy of the liquidityconstrained true innovator. In the low-imitation-cost case, $\bar{n}\left(I_{2}\right)$ decreases and, as a result, the number of false innovator applications falls.

In the high-imitation-cost case, the rise in $\theta$ affects the mixed strategies of the false type. When the probability of correct screening increases with the scrutiny of the PTO, a rising $\theta$ entails the same effects on $h\left(\bar{n}\left(I_{2}\right)\right)$, as is the case in Corollary 2. Hence, the same condition guarantees that the expected number of applications by the false innovator decreases with $\theta$. This leads us to the following proposition:

Proposition 3 Suppose that patenting fees are a weakly increasing function of the PTO's accuracy, $d P / d \theta \geq 0$. In this case, if "the probability of correct screening", $g\left(\theta, \bar{n}\left(I_{2}\right)\right)$, increases with $\theta$, then:

i) in the low-imitation-cost case, an increase in $\theta$ always leads to a decrease in the overall number of applications;

ii) in the high-imitation-cost case, condition (2) is sufficient to guarantee that the overall number of applications decreases as $\theta$ gets larger.

Proposition 3 highlights the conditions under which all three types of innovator file a smaller number of applications as $\theta$ rises. These results are complemented by the analysis in Section 5.1 below.

\subsection{Two-tiered patent system}

Another proposal for mitigating the proliferation of bad patents is to introduce a two-tiered system based on two patent types: a "gold-plate" patent entailing larger patenting fees, stricter scrutiny by the PTO and providing stronger protection when awarded, and a "regular" patent requiring lower fees and less scrutiny, but granting weaker rights to the patentee (see Atal and Bar, 2014).

What we argue below is that a two-tiered patent system is likely to be of little use once we depart from the standard assumption that the firm can only choose whether to apply for one or none patents. When the firm can also select the number of applications to file it can endogenously gold-plate its innovation by increasing $n$. Hence having two types of patents is redundant.

Consider the following setting. There are two types of patents: a regular patent with fees $P_{r}$ and accuracy $\theta_{r}$ and a gold-plate patent with fees $P_{g}(>$ $\left.P_{r}\right)$ and accuracy $\theta_{g}\left(>\theta_{r}\right)$. For the sake of simplicity, we assume that the firm can only choose one type of patents, i.e. all applications filed by the 
firm must belong to the same tier. Moreover, we suppose that Assumption 2 applies both to gold as well as to regular patents.

Let $x$ be the amount of resources the firm spends in patenting i.e. $x=n P$; with a slight change in notation we let $g\left(\theta_{i}, x\right)$ be the probability of the PTO detecting the false innovator when the firm spends $x>0$ in applying for type $i=r, g$ patents. Without loss of generality, we assume that:

Assumption $3 g\left(\theta_{g}, x\right)=g\left(\theta_{r}, x\right)$, for all $x>0$.

Assumption 3 implies that if the firm spends the same amount of money on patenting, the probability of detecting the false innovator is the same irrespective of whether the firm applies for regular or gold-plate patents. In other words, the two tiers are "equally efficient" in revealing information on the firm type.

Proposition 4 compares a single-tier patent system (for instance composed of regular patents only) and a two-tiered patent system.

Proposition 4 The equilibrium of the patenting game with a two-tiered patent system is equivalent to that with a single tier.

In the Appendix we show that the equilibrium of the patenting game that one obtains with a single or with a two-tiered patent system is payoff equivalent. Hence, adding a second tier does not alter the market outcome whatsoever.

Proposition 4 is based on the assumption that the two tiers are equally efficient (Assumption 3). However, the result can be easily generalized; it would be straightforward to show that if one tier is more efficient than the other (e.g. the gold-plate patent is more efficient: $g\left(\theta_{g}, x\right)>g\left(\theta_{r}, x\right)$ for any $x>0$ ), then in equilibrium the firm would never select the less efficient tier which therefore would play no role at all.

\subsection{Penalties/patent bounties}

One possible way to reduce the number of bad patents is by fining the applicant should the PTO reject the application. Fines or penalties may take the form of, for instance, patent bounties (see Thomas, 2001). Clearly, in the setting we are analyzing penalties would alleviate the bad patents problem. We are assuming that the PTO does not commit type II errors (applications filed by the true types are always accepted), hence only applications by the false type are rejected. Therefore, as the amount of fines increases, the incentives for the false innovator to file patent applications diminishes. 
The situation changes radically when there is even a small probability that the PTO commits type II errors (we address this case in Section 5.2). In this case penalties are also imposed on the true types, and this may reduce the ability of the liquidity-constrained true innovator to signal its type. If the PTO imposes penalties in addition to patenting fees $P$, then the budget for patenting shrinks thus reducing the informativeness of the signal sent through the PTO. Substituting patenting fees $P$ with penalties (i.e. having a PTO that only finances itself through the fees it collects when rejecting patent applications) may not work either. Besides distorting the behavior of the PTO which would become more prone to rejecting applications, it might substantially reduce the number of patents filed by liquidity-constrained true innovators. When choosing how many patent applications to file firms need to consider the "worst case scenario" in which many of their applications are rejected. Hence, in order to be able to cope with (potentially) very large penalties, firms may need to reduce the number of applications they file substantially, thus decreasing, once again, the informativeness of the signal sent by the PTO.

\section{Extensions}

In this section, we analyze the robustness of our findings to some extensions to the basic model developed above. In order to simplify the exposition, we restrict our attention to the low-imitation-cost case in which the false type chooses $\bar{n}\left(I_{2}\right)$ with probability one.

\section{$5.1 \quad$ R\&D incentives}

In the previous sections, we assumed that the true types are able to collect profits $l$-times larger than those of the false innovator. A natural way of endogenizing $l$ is by assuming that, in addition to the investment decision, the firm chooses an R\&D effort which is only productive if the project is completed, and is worthless if the firm becomes a false innovator.

Suppose that the firm starts working on an idea for a research project while there is still uncertainty about its financial viability. Formally, the firm chooses an effort level $e \geq 0$ at time $t=0$, before knowing the size of the investment needed to complete the project, that is, before time $t=1$ when the amount of the investment $i \in\left\{I_{1}, I_{2}, I_{3}\right\}$ is observed. Effort is exerted at cost $c(e) \geq 0$, with $c^{\prime}(e)>0$ and $c^{\prime \prime}(e) \geq 0$. We assume that $c(e)$ is a non-monetary cost, so that the effort decision does not affect the budget for the investment and the patenting stages. As anticipated above, effort only 
increases revenues when innovation is actually developed; otherwise it is lost. Moreover, we assume that: $l(e) \geq 1$, with $l^{\prime}(e)>0$ and $l^{\prime \prime}(e)<0$. Finally, for the sake of simplicity, the probabilities $p_{i}, i=1,2,3$, are assumed to be independent from effort.

When choosing $e$ the firm anticipates that:

- with probability $p_{1}$ it will observe $i=I_{1}$ and therefore: it will become a deep-pocket true innovator that applies for $n^{T}$ patents, separates and obtains $f(1) \Delta l(e)+r l(e)+K-n^{T} P \equiv \pi_{1}$;

- with probability $p_{2}$ it will observe $i=I_{2}$ and therefore: it will become a liquidity-constrained true innovator that spends its entire budget filing $\bar{n}\left(I_{2}\right)$ applications, that is unable to separate from the false innovator, and obtains $f\left(\xi_{\bar{n}\left(I_{2}\right)}\right) \Delta l(e)+r l(e) \equiv \pi_{2}$;

- with probability $p_{3}$ it will observe $i=I_{3}$ and therefore: it will become a false innovator that applies for $\bar{n}\left(I_{2}\right)$ patents and obtains

$$
g\left(\theta, \bar{n}\left(I_{2}\right)\right)(f(0) \Delta+r)+\left(1-g\left(\theta, \bar{n}\left(I_{2}\right)\right)\right)\left(f\left(\xi_{\bar{n}\left(I_{2}\right)}\right) \Delta+r\right)+K-\bar{n}\left(I_{2}\right) P \equiv \pi_{3} .
$$

Hence, the firm chooses $e$ in order to:

$$
\max _{e} p_{1} \pi_{1}+p_{2} \pi_{2}+p_{3} \pi_{3}-c(e)
$$

By implicitly differentiating the first order condition of the maximization problem above, it is possible to show that:

Proposition 5 Suppose that at $t=0$, before observing the realization of $i$, the firm chooses an effort e which only increases the profits of true types. In the low-imitation-cost case, the effort chosen by the firm increases with $\theta$ if and only if "the probability of correct screening", $g\left(\theta, \bar{n}\left(I_{2}\right)\right)$, rises with $\theta$.

The effect of raising the bar on the incentives to exert R\&D efforts critically depends on how $\theta$ impacts on $g\left(\theta, \bar{n}\left(I_{2}\right)\right)$. When the probability of correct screening increases with $\theta$, the PTO's decisions become more informative and this benefits the liquidity-constrained true innovator who is unable to separate. An increase in $g\left(\theta, \bar{n}\left(I_{2}\right)\right)$ enlarges $\xi_{\bar{n}\left(I_{2}\right)}$ and therefore $\pi_{2}$, thus providing stronger incentives to exert effort.

It is worth noting two implications of this result. When the patenting fees are not affected by $\theta$, a tighter PTO scrutiny certainly increases the precision of the information revealed by the PTO's decisions, thus enhancing R\&D incentives. The opposite result emerges when the PTO increases fees 
without making the examination process more stringent (i.e. when $P$ is increased while $\theta$ is left unchanged). In this case, the probability of correct screening falls, since the only effect of the policy change is the reduction of $\bar{n}\left(I_{2}\right)$. As a consequence, an increase in patenting fees without tougher scrutiny would reduce the effort incentives.

\subsection{The PTO's technology}

So far, we have assumed that the PTO only commits type I errors (it grants patents to the false innovator with positive probability) and "sends" just two types of signals. As a matter of fact, when rejecting some applications, the PTO certifies that the firm is the false innovator and consequently sends a fully informative signal. By contrast, whenever it accepts all the applications that have been filed, the PTO sends a partially informative signal: the probability that the firm is a true type is larger than the priors, given that the PTO has failed to detect the false innovator. The aim of this section is to prove that the equilibrium characterized in Proposition 1 is still an equilibrium even when we assume a more sophisticated PTO's "technology". In particular, we now consider a setting in which the PTO commits both type I and type II errors (it rejects applications filed by the true types with positive probability) and sends a richer set of signals than we have considered so far.

As usual, $n$ is the number of applications, while we let $m$ denote the number of granted patents, and $n-m$ the number of applications that are rejected by the PTO. Both $n$ and $m$ are continuous variables, with $n \geq 0$ and $m \in[0, n]$. While $n$ is chosen by the firm, $m$, on the other hand, is determined probabilistically. The probability that the PTO grants $m$ patents out of $n$ applications depends on whether the applicant is a true or a false innovator. Formally, we let $\phi_{T}(m / n)$ and $\phi_{F}(m / n)$ be the probability density functions when the applicant is the true or the false type respectively and, in what follows, we assume them to be beta functions with shape parameters $(1,1)$ and $(1,2)$ respectively. Clearly, $\phi_{T}(m / n)$ and $\phi_{F}(m / n)$ are re-scaled in order for $m$ to take values on the whole $[0, n]$ support. In other words, $\phi_{T}(m / n)=1 / n, \phi_{F}(m / n)=2(n-m) / n^{2}$ and the support in both cases is the segment $[0, n]$.

The density functions we are working with give rise to a sensible system of beliefs. Suppose that only the false innovator and the liquidity-constrained true innovator choose $n$ and that they do so with probability 1 . The belief conditional on observing $m$ patents being granted by the PTO (and $n-m$ rejected) is the following: 


$$
\xi_{n}(m)=\frac{n p_{2}}{n p_{2}+2 p_{3}(n-m)} .
$$

As argued above, the set of information that the PTO's decisions reveal is much richer than the one we considered in the previous sections. Indeed, each realization of $m \in[0, n]$ gives rise to a different belief. Moreover, $\xi_{n}(m)$ is such that: $i$ ) the decision of the PTO is never fully informative as regards the firm type (any $m \in[0, n]$ might realize when the firm is either the true or the false type); ii) $\xi_{n}(m)$ increases in $m$, so that for a given number $n$ of applications the larger the number of granted patents the larger the probability that the firm is a true type; iii) $\xi_{n}(m)$ decreases in $n$, so that for a given number $m$ of granted patents the higher the number of applications the lower the probability that the firm is a true innovator.

For the sake of simplicity we assume that $p_{2}=p_{3}$ and that $f(\xi)=\xi$. Under these assumptions we are able to prove the following result.

Proposition 6 Suppose that the PTO grants $m$ patents out of $n$ applications and rejects the remaining $n-m$ according to the probability density functions $\phi_{T}(m / n)=1 / n$ and $\phi_{F}(m / n)=2(n-m) / n^{2}$. If $\bar{n}\left(I_{2}\right) P<(1-(\ln 3) / 2) \Delta$ and $l>(\ln 3) /(2-\ln 3)$, the equilibrium shown in Proposition 1 for the low-imitation-cost case is still an equilibrium of the patenting stage in this modified setting.

In this modified setting, the same equilibrium arises as in the low-imitationcost case of Proposition 1 provided that $\bar{n}\left(I_{2}\right) P$ is low enough and $l$ is sufficiently large. The condition on $\bar{n}\left(I_{2}\right) P$ corresponds to the condition discussed in the comment to the low-imitation-cost case of Proposition 1 and, clearly, the interpretation is similar. The condition on $l$ implies that profits for the true types are significantly larger than those for the false innovator. In particular, it implies that the deep-pocket true innovator prefers to separate by choosing $n^{T}$. Note that the condition on $l$ is more restrictive than the equivalent condition in the basic model $(l \geq 1)$. This is due to the fact that, in the new setting, the false innovator is never "certified" as false, and this in turn makes mimicking the true type a less risky strategy.

\subsection{Equilibrium selection criterion}

In the paper, we have employed the D1 criterion to refine the equilibrium at the patenting stage. As a result, we have been able to select a unique equi-

librium for the signalling game. Given the sensitivity of equilibrium selection to the choice of the refinements, in this section we discuss what the results 
would have been were we to employ the more standard intuitive criterion (Cho and Kreps, 1987). In particular, we claim that our results would have been qualitatively the same. To see this, first of all note that Lemma 3 is valid for any PBE and does not hinge upon the D1 criterion; hence, the false type does not separate. Let us now consider the deep-pocket true innovator. For this firm type, the intuitive criterion plays the same role as in standard signalling models á la Spence: the deep-pocket true innovator separates by applying for a number of patents $\tilde{n}^{T}$ that makes indifferent the false type between choosing $\tilde{n}^{T}$ and playing the equilibrium strategy. This result follows from the fact that the intuitive criterion imposes the belief $\xi=1$ if $n \geq \tilde{n}^{T}$ is observed. By contrast, the intuitive criterion is silent when $n<\tilde{n}^{T}$ is chosen out-of-the-equilibrium path. Hence, the false innovator and the liquidityconstrained true innovator choose the same number of applications (Lemma 3 applies), though this is not necessarily equal to $\bar{n}\left(I_{2}\right)$. In other words, in equilibrium, the false innovator and the liquidity-constrained true innovator pool by choosing $n^{\text {pool }}$, with $n^{\text {pool }} \in\left[0, \bar{n}\left(I_{2}\right)\right]$. This choice is supported by the following out-of-equilibrium beliefs: $\xi=0$ if $n$ applications are filed, with $n \neq n^{p o o l}$ and $n<\tilde{n}^{T}$.

In summary, if we were to employ the intuitive criterion, the equilibrium described in Proposition 1 would survive but other equilibria might emerge. However, all these equilibria share the same distinctive feature: the false innovator and the liquidity-constrained true innovator apply for the same number of patents, $n^{\text {pool }} \in\left[0, \bar{n}\left(I_{2}\right)\right]$, while the deep-pockets true innovator files the minimum number of applications that the false type is not willing to apply for. Hence, the only difference that emerges by using the intuitive criterion instead of D1 is that the equilibrium described in Section 3 is not unique.

\subsection{Patenting for protective reasons}

An important function of patents is to protect innovations from imitation. A possible way of incorporating this further effect in our model is to assume that the profits for the true types are an increasing function of the number of patents protecting the innovation; that is, $l(n)$ with $l^{\prime}(n) \geq 0 .{ }^{20}$ Clearly, this assumption raises the patenting incentives for the true innovators and, for this reason, it would be easy to verify that Lemmas 1 through 4 are still valid, given that they require the true types to apply for a large number of

\footnotetext{
${ }^{20}$ The assumption that only the true innovators benefit from patent protection seems consistent with our model in which only the true types have actually innovated. A milder assumption delivering the same results would be that the true types benefit more than the false innovator from patent protection.
} 
patents. Hence, the liquidity-constrained true innovator and the false type would still choose $\bar{n}\left(I_{2}\right)$.

Moreover, the behavior of the deep-pocket true innovator would be unaffected unless the protective reasons for patenting were so strong as to induce this firm type to increase its number of applications above the level that ensures separation from the false innovator (i.e. applying for a number of patents above $n^{T}$ ). Clearly, in this latter case, an increase in $\theta$ would affect neither the behavior of the deep-pocket true innovator nor the overall number of applications.

\subsection{Other sources of information}

In the paper, we have implicitly assumed that the market only obtains information about the firm type through the decisions of the PTO (or through the strategies played by the different firm types). This assumption, which focuses on the importance of the PTO's decisions in revealing information, is grounded on the empirical evidence showing that, for instance, the size of the patent portfolio increases the firm's chances of being financed by a venture capitalist (see our discussion in the Introduction). However, in some cases, it is reasonable to assume that the market can obtain information about the firm type directly from the patent application, e.g. by reading the description and the claims that applications contain a venture capitalist might learn useful details about the firm.

A possible way of dealing with this additional source of information is to assume that, in order to infer firm type, the market uses both the information revealed by the PTO's decisions as well as its own ability to read and understand the applications. For instance, the overall probability of detecting the false type could be given by $G(\alpha, \theta, n)=g(\theta, n)+\alpha n$, so that an increase in the number of applications would improve the chances of the false innovator being detected either through the "PTO channel" $(g(\theta, n))$, or by means of the information the market is able to infer on its own $(\alpha n)$. The information collected through this latter channel might be affected by the sector in which the firm is operating (e.g. $\alpha$ is large in sectors where the information contained in the patent applications is particularly revealing) or, more generally, by the ability of the market to interpret the information contained in the patent applications.

In such a setting, $\alpha$ and $\theta$ play a similar role: an increase in either $\alpha$, $\theta$ or both enhances the chances of detecting the false innovator. Hence, the consequences of the additional source of information $\alpha n$ can be figured out by means of the comparative statics results derived in the paper. From this observation, it follows that an increase in $\alpha$ reduces the number of patents 
necessary in order to separate from the false innovator, and that for large values of $\alpha$ a small number of applications might allow true innovators to signal their type. To sum up, the presence of an additional source of information obviously reduces the importance of the PTO in revealing information. However, as argued above, empirical evidence suggests that the PTO's decision conveys substantially valuable information for innovators.

\section{Conclusions}

Our paper offers a novel explanation to the recent surge in patenting; the argument is based on two key facts. First, empirical evidence shows that patents play a crucial signaling role and mitigate the asymmetry of information between start-up companies and external investors. Second, during the last few years, PTOs have lowered their standards and, as a result, have granted an increasing number of bad patents.

In this context, we have shown that firms have great incentives to apply for patents and they file a higher number of applications than they would choose to if the PTO had a better screening ability. Poor screening by the PTOs offers "false innovators" a greater chance of being granted patents, giving them incentives to file applications. Interestingly, "true innovators" are consequently induced to patent even more intensively: they do so in order to separate from false innovators. However, if an innovating firm is liquidity-constrained it may be unable to apply for a sufficiently large number of patents to credibly signal its type. In an extension of the model we have shown that the absence of a credible signal due to poor PTO screening reduces a firm's expected revenues and $R \& D$ incentives.

An interesting implication of our paper is that the firm type is revealed either by the number of applications that are filed or by the number of patents granted (i.e. by the PTO's decision), depending on whether the true innovator is able to separate or not. This result reconciles some apparently contradictory findings in the empirical literature. As a matter of fact, while some studies show that awarded patents do not increase the chances of receiving financial aid (e.g. Haüssler et al., 2009), others report that the PTO decision to grant a patent further enhances the likelihood of the company being financed (e.g. Greenberg, 2013).

In Section 4, we also considered some policy interventions that have been proposed to reform the patent system and cope with the bad patents issue. Based on our model, we provide an intuitive condition under which a tightening of the patentability standards ("raising the bar"), possibly in conjunction with an increase in the patenting fees, is likely to mitigate the bad patents 
problem. Finally, we show that the proposal to introduce a two-tiered patent system is unlikely to be effective, once we depart from the standard assumption that firms can apply for either zero or one patent. Indeed, firms can "gold-plate" their innovations simply by increasing the number of applications they file, making the second patent tier redundant.

Generally speaking, from a policy perspective, our model suggests that patent reform proposals should carefully consider the effects of the Patent Offices screening process on the signaling role played by patents. This is particularly important for startups and young companies that rely more strongly on patents as signaling devices.

\section{Appendix (1): proofs}

\section{Proof of Lemma 1}

Three cases need to be considered in order to prove Lemma 1. Case i): $n+\varepsilon$ is an equilibrium choice of the true types only. In this case the equilibrium belief associated with strategies $n$ and $n+\varepsilon$ are $\xi_{n}<1$ and $\xi_{n+\varepsilon}=1$ respectively; therefore, for $\varepsilon$ positive but negligible the true innovator certainly prefers $n+\varepsilon$ to $n$.

Case ii): $n+\varepsilon$ is an equilibrium choice also of the false innovator. In this case, the false innovator has to be indifferent between $n+\varepsilon$ and $n$. Hence, the payoff associated with the strategy $n+\varepsilon(g(\theta, n+\varepsilon) f(0) \Delta+(1-g(\theta, n+$ $\left.\varepsilon)) f\left(\xi_{n+\varepsilon}\right) \Delta+r+K-(n+\varepsilon) P\right)$ has to be the same as that associated with strategy $n\left(g(\theta, n) f(0) \Delta+(1-g(\theta, n)) f\left(\xi_{n}\right) \Delta+r+K-n P\right)$; this occurs when $\varepsilon P=\Gamma \Delta$, where:

$$
\Gamma \equiv(1-g(\theta, n+\varepsilon))\left[f\left(\xi_{n+\varepsilon}\right)-f\left(\xi_{n}\right)\right]-(g(\theta, n+\varepsilon)-g(\theta, n))\left[f\left(\xi_{n}\right)-f(0)\right]
$$

Notice that since $\partial g(\theta, n) / \partial n>0$ and $f^{\prime}(\xi)>0$, then $\varepsilon P=\Gamma \Delta$ implies that $\xi_{n+\varepsilon}>\xi_{n}$. Next we show that when condition $\varepsilon P=\Gamma \Delta$ is satisfied, then the true types strictly prefer strategy $n+\varepsilon$ (with associated payoff $f\left(\xi_{n+\varepsilon}\right) \Delta l+r l+K-(n+\varepsilon) P$ ) to $n$ (with associated payoff $f\left(\xi_{n}\right) \Delta l+r l+$ $K-n P)$. By comparing the two payoffs it follows that the true types prefer $n+\varepsilon$ to $n$ whenever $\varepsilon P<\chi \Delta l$, where $\chi \equiv f\left(\xi_{n+\varepsilon}\right)-f\left(\xi_{n}\right)$.

Notice that $\Delta l \geq \Delta$ (since $l \geq 1)$ and $\chi>\Gamma$ since $g(\theta, n+\varepsilon)\left[f\left(\xi_{n+\varepsilon}\right)-\right.$ $f(0)]>g(\theta, n)\left[f\left(\xi_{n}\right)-f(0)\right]$ which in turn is verified given that $\partial g(\theta, n) / \partial n>$ $0, f^{\prime}(\xi)>0$ and $\xi_{n+\varepsilon}>\xi_{n}$. Therefore, we have shown that $\varepsilon P<\chi \Delta l$ when $\varepsilon P=\Gamma \Delta$ which implies that when the false innovator is indifferent between $n+\varepsilon$ and $n$, then the true types strictly prefer $n+\varepsilon$ to $n$.

Case iii): $n+\varepsilon$ is an out of equilibrium choice. By using the previous computations, it follows that the true types prefer $n+\varepsilon$ to $n$ provided that 
the out-of-equilibrium belief $\xi_{n+\varepsilon}$ is such that $\varepsilon P<\chi \Delta l$; in turn, the false innovator prefers $n+\varepsilon$ to $n$ iff $\varepsilon P<\Gamma \Delta$. Since $\Delta l \geq \Delta$ and $\chi>\Gamma$ as argued above, then condition $\varepsilon P<\Gamma \Delta$ implies $\varepsilon P<\chi \Delta l$ while the reverse implication does not hold. Therefore, according to the D1 criterion the out of equilibrium belief associated with $n+\varepsilon$ is $\xi_{n+\varepsilon}=1$; in turn, this belief implies that the true types prefer $n+\varepsilon$ to $n$.

\section{Proof of Lemma 2}

Follows directly from Lemma 1 and Assumption 2-i).

\section{Proof of Lemma 3}

We prove the lemma by contradiction. Suppose that the false innovator separates with probability 1 ; then it certainly chooses $n=0$, thus obtaining $f(0) \Delta+r+K$. In turn, the liquidity-constrained true innovator chooses some $0<n^{\prime} \leq \bar{n}\left(I_{2}\right)$. By deviating and choosing $n^{\prime}$, in a separating equilibrium, the false type obtains $g\left(\theta, n^{\prime}\right) f(0) \Delta+\left(1-g\left(\theta, n^{\prime}\right)\right) f(1) \Delta+r+K-n^{\prime} P$. One can easily check that under assumption 2-ii) the false innovator benefits from deviating and choosing $n^{\prime}$ for any $0<n^{\prime} \leq \bar{n}\left(I_{2}\right)$.

\section{Proof of Lemma 4}

We prove the lemma by contradiction. Suppose that the liquidity-constrained true innovator chooses $n<\bar{n}\left(I_{2}\right)$ with some positive probability. Two cases are possible. Case i): also the false innovator chooses $n$ with some positive probability. In this case, from Lemma 1 we know that the liquidityconstrained true innovator prefers to deviate and choose $n+\varepsilon$ rather than $n$; hence, we have a contradiction.

Case ii): $n$ is not chosen by the false innovator, which implies that the associated belief is $\xi_{n}=1$. Two subcases are possible: ii-a) the false type chooses some $n^{\prime}<n$ selected also by the liquidity-constrained true innovator (the deep-pocket true innovator separates, as shown in Lemma 2); or ii-b) the false innovator chooses $n=0$ and separates. Subcase ii-a) is not possible since, by Lemma 1, the liquidity-constrained true innovator prefers (and can) deviate by playing $n^{\prime}+\varepsilon$. Subcase ii-b) is not possible either: the false innovator separating with probability 1 violates Lemma 3.

\section{Proof of Proposition 1}

Consider the false innovator and let $n^{T}$ be the number of applications that makes this firm type indifferent between $\bar{n}\left(I_{2}\right)$ and $n^{T}$ provided that: i) the market holds the belief $\xi_{n^{T}}=1$ when the event " $n^{T}$ is chosen and the PTO does not certify the firm to be the false type" occurs, ii) the market holds the belief $\xi_{\bar{n}\left(I_{2}\right)}=p_{2} /\left[p_{2}+p_{3}\left(1-g\left(\theta, \bar{n}\left(I_{2}\right)\right)\right) h\left(\bar{n}\left(I_{2}\right)\right)\right]$ (see the statement of the proposition) when the event " $\bar{n}\left(I_{2}\right)$ is chosen and the PTO does not certify 
the firm to be the false type" occurs. In other words, $n^{T}$ is the value of $n$ that satisfies the following equality

$$
\begin{gathered}
g\left(\theta, \bar{n}\left(I_{2}\right)\right) f(0) \Delta+\left(1-g\left(\theta, \bar{n}\left(I_{2}\right)\right)\right) f\left(\xi_{\bar{n}\left(I_{2}\right)}\right) \Delta+r+K-\bar{n}\left(I_{2}\right) P= \\
g(\theta, n) f(0) \Delta+(1-g(\theta, n)) f(1) \Delta+r+K-n P .
\end{gathered}
$$

Notice that $n^{T}>\bar{n}\left(I_{2}\right)$ otherwise the above equality cannot hold.

Clearly, the false innovator prefers $\bar{n}\left(I_{2}\right)$ to any $n>n^{T}$ and it is indifferent between $\bar{n}\left(I_{2}\right)$ and $n^{T}$. Moreover, given the equilibrium beliefs, it prefers $n=0$ to any $n \in\left(0, \bar{n}\left(I_{2}\right)\right)$ and to any $n \in\left(\bar{n}\left(I_{2}\right), n^{T}\right)$. Hence, the only possible choices of the false innovator are either $\bar{n}\left(I_{2}\right)$ or $n=0$; this latter strategy yields a payoff equal to $f(0) \Delta+r+K$.

Notice that:

a) $\xi_{\bar{n}\left(I_{2}\right)}=p_{2} /\left[p_{2}+p_{3}\left(1-g\left(\theta, \bar{n}\left(I_{2}\right)\right)\right) h\left(\bar{n}\left(I_{2}\right)\right)\right]$ is a decreasing function of $h\left(\bar{n}\left(I_{2}\right)\right)$; then, the payoff associated with the strategy $\bar{n}\left(I_{2}\right)$ decreases as $h\left(\bar{n}\left(I_{2}\right)\right.$ increases;

b) under Assumption 1-ii), when $h\left(\bar{n}\left(I_{2}\right)\right)=0$ the payoff associated with strategy $\bar{n}\left(I_{2}\right)$ is larger than the payoff associated with strategy $n=0$;

c) when $h\left(\bar{n}\left(I_{2}\right)\right)=1$, then $\xi_{\bar{n}\left(I_{2}\right)}=p_{2} /\left[p_{2}+p_{3}\left(1-g\left(\theta, \bar{n}\left(I_{2}\right)\right)\right)\right]$ and $\bar{n}\left(I_{2}\right)$ is preferred to $n=0$ by the false type provided that $\bar{n}\left(I_{2}\right) P \leq(1-$ $\left.\left.g\left(\theta, \bar{n}\left(I_{2}\right)\right)\right)\left(f\left(p_{2} /\left[p_{2}+p_{3}\left(1-g\left(\theta, \bar{n}\left(I_{2}\right)\right)\right)\right]\right)\right)-f(0)\right) \Delta$.

Summarizing, the optimal behavior of the false type is:

- when $\left.\bar{n}\left(I_{2}\right) P \leq\left(1-g\left(\theta, \bar{n}\left(I_{2}\right)\right)\right)\left(f\left(p_{2} /\left[p_{2}+p_{3}\left(1-g\left(\theta, \bar{n}\left(I_{2}\right)\right)\right)\right]\right)\right)-f(0)\right) \Delta$, it chooses $\bar{n}\left(I_{2}\right)$ with probability $h\left(\bar{n}\left(I_{2}\right)\right)=1$;

- when $\bar{n}\left(I_{2}\right) P \in\left(\left(1-g\left(\theta, \bar{n}\left(I_{2}\right)\right)\right)\left(f\left(p_{2} /\left[p_{2}+p_{3}\left(1-g\left(\theta, \bar{n}\left(I_{2}\right)\right)\right)\right]\right)-\right.\right.$ $\left.f(0)) \Delta,\left(1-g\left(\theta, \bar{n}\left(I_{2}\right)\right)\right)(f(1)-f(0)) \Delta\right)$, it chooses $\bar{n}\left(I_{2}\right)$ with probability $h\left(\bar{n}\left(I_{2}\right)\right) \in(0,1)$ and $n=0$ with complementary probability. The probability $h\left(\bar{n}\left(I_{2}\right)\right) \in(0,1)$ is such that the false type is indifferent between $\bar{n}\left(I_{2}\right)$ and $n=0$; from a), b) and c) above such $h\left(\bar{n}\left(I_{2}\right)\right)$ exists.

Consider the deep-pocket true innovator; given the equilibrium beliefs, it prefers $n^{T}$ to any $n>n^{T}$. Moreover, by using the same arguments as those shown in Lemma 1, it would be easy to verify that it prefers $n^{T}$ to any $n<n^{T}$ with $n \neq \bar{n}\left(I_{2}\right)$ since the false does so. Similarly, the deep-pocket true innovator prefers $n^{T}$ to $\bar{n}\left(I_{2}\right)$, given that the false type is indifferent between the two choices. Finally, the optimal choice of the liquidity-constrained true innovator is $\bar{n}\left(I_{2}\right)$, as shown by Lemma 4 .

Consider now the beliefs. Since $n=n^{T}$ is chosen by the deep-pocket true innovator only, then it follows that $\xi_{n^{T}}=1$. From the definition of $n^{T}$, the false innovator never benefits from choosing $n>n^{T}$, therefore $\xi_{n}=1$ is associated with any $n>n^{T}$. When $n=\bar{n}\left(I_{2}\right)$ is chosen and the PTO does 
not certify the firm to be the false innovator, the belief $\xi_{\bar{n}\left(I_{2}\right)}=p_{2} /\left[p_{2}+\right.$ $\left.p_{3}\left(1-g\left(\theta, \bar{n}\left(I_{2}\right)\right)\right) h\left(\bar{n}\left(I_{2}\right)\right)\right]$ follows from Bayes rule. Consider now an outof-equilibrium choice $n \in\left(\bar{n}\left(I_{2}\right), n^{T}\right)$. Clearly, the liquidity-constrained true innovator cannot apply for such a large number of patents. The deep-pocket true innovator prefers $n \in\left(\bar{n}\left(I_{2}\right), n^{T}\right)$ to the equilibrium strategy $n^{T}$ if and only if $\xi_{n}$, the out-of-equilibrium belief associated with the event " $n$ is chosen and the PTO does not certify the firm to be the false innovator", is such that

$$
\begin{gathered}
f\left(\xi_{n}\right) \Delta l+r l+K-n P \geq f(1) \Delta l+r l+K-n^{T} P \\
\Leftrightarrow\left(n^{T}-n\right) P \geq\left(f(1)-f\left(\xi_{n}\right)\right) \Delta l
\end{gathered}
$$

In turn, the false type prefers $n \in\left(\bar{n}\left(I_{2}\right), n^{T}\right)$ to the equilibrium strategy $\bar{n}\left(I_{2}\right)$ whenever $\xi_{n}$ is such that (notice that here we exploit the fact that the false innovator is indifferent between $\bar{n}\left(I_{2}\right)$ and $n^{T}$ )

$$
\begin{aligned}
& g(\theta, n) f(0) \Delta+(1-g(\theta, n)) f\left(\xi_{n}\right) \Delta+r+K-n P \geq g\left(\theta, n^{T}\right) f(0) \Delta+\left(1-g\left(\theta, n^{T}\right)\right) f(1) \Delta+r+K-n^{T} P \\
& \Leftrightarrow\left(n^{T}-n\right) P \geq\left(1-g\left(\theta, n^{T}\right)\right)\left[f(1)-f\left(\xi_{n}\right)\right]-\left(g\left(\theta, n^{T}\right)-g(\theta, n)\right)\left[f\left(\xi_{n}\right)-f(0)\right](5)
\end{aligned}
$$

A simple comparison between conditions (4) and (5) implies that whenever the former one is satisfied also latter holds, while the reverse is not true. Therefore, the D1 criterion implies that the belief $\xi_{n}=0$ must be associated with the out-of-equilibrium choice $n \in\left(\bar{n}\left(I_{2}\right), n^{T}\right)$.

The same arguments can be used to show that for all the out-of-equilibrium beliefs such that the liquidity-constrained true innovator prefers $n \in\left(0, \bar{n}\left(I_{2}\right)\right)$ to the equilibrium strategy $\bar{n}\left(I_{2}\right)$ also the false innovator does so while the reverse is not true. Hence, the D1 criterion implies that the belief $\xi_{n}=0$ must be associated with the out-of-equilibrium choice $n \in\left(0, \bar{n}\left(I_{2}\right)\right.$ ) (notice that the deep-pocket true innovator obtains a higher payoff from $n^{T}$ than from $\left.\bar{n}\left(I_{2}\right)\right)$. Finally, the belief $\xi_{n=0}=0$ is associated with the choice $n=0$ given that: i) if $h\left(\bar{n}\left(I_{2}\right)\right) \in(0,1)$ it follows from Bayes' rule; ii) if $h\left(\bar{n}\left(I_{2}\right)\right)=1$, condition $\bar{n}\left(I_{2}\right) P \leq\left(1-g\left(\theta, \bar{n}\left(I_{2}\right)\right)\right)\left(f\left(p_{2} /\left[p_{2}+p_{3}\left(1-g\left(\theta, \bar{n}\left(I_{2}\right)\right)\right)\right]\right)-f(0)\right) \Delta$ implies that the choice $n=0$ is equilibrium dominated for all three types of the firm; hence the belief $\xi_{n=0}=0$ is consistent with the D1 criterion.

\section{Proof of Corollaries 1 and 2}

Particular cases of Propositions 2 and 3 respectively. 


\section{Proof of Proposition 2}

We start the proof by focussing on the low-imitation-cost case. Consider first the behavior of the deep-pocket true innovator. In equilibrium, it applies for a number of patents $n^{T}$ defined as the value of $n$ that makes the false type indifferent between choosing $\bar{n}\left(I_{2}\right)$ and $n$; namely

$$
\begin{aligned}
& g\left(\theta, \bar{n}\left(I_{2}\right)\right) f(0) \Delta+\left(1-g\left(\theta, \bar{n}\left(I_{2}\right)\right)\right) f\left(\xi_{\bar{n}\left(I_{2}\right)}\right) \Delta+r+K-\bar{n}\left(I_{2}\right) P- \\
& {[g(\theta, n) f(0) \Delta+(1-g(\theta, n)) f(1) \Delta+r+K-n P]=0 . }
\end{aligned}
$$

Denote by $(\cdot)$ the above expression and recall that $K-\bar{n}\left(I_{2}\right) P=I_{2}$. From the implicit function theorem it follows that $d n^{T} / d \theta=-(\partial(\cdot) / \partial \theta) /(\partial(\cdot) / \partial n)$.

$$
\begin{aligned}
\frac{\partial(\cdot)}{\partial \theta}= & \frac{\partial g\left(\theta, \bar{n}\left(I_{2}\right)\right)}{\partial \theta}\left(f(0)-f\left(\xi_{\bar{n}\left(I_{2}\right)}\right)\right) \Delta-\frac{\partial g\left(\theta, n^{T}\right)}{\partial \theta}(f(0)-f(1)) \Delta+ \\
& \frac{\partial g\left(\theta, \bar{n}\left(I_{2}\right)\right.}{\partial n} \frac{\partial \bar{n}\left(I_{2}\right)}{\partial P} \frac{d P}{d \theta}\left(f(0)-f\left(\xi_{\bar{n}\left(I_{2}\right)}\right)\right) \Delta+n \frac{d P}{d \theta} \\
+ & \left(1-g\left(\theta, \bar{n}\left(I_{2}\right)\right)\right) \frac{d f}{d \xi} \frac{\partial \xi}{\partial g}\left[\frac{\partial g\left(\theta, \bar{n}\left(I_{2}\right)\right)}{\partial \theta}+\frac{\partial g\left(\theta, \bar{n}\left(I_{2}\right)\right)}{\partial n} \frac{\partial \bar{n}\left(I_{2}\right)}{\partial P} \frac{d P}{d \theta}\right]
\end{aligned}
$$

The sum of the first two terms is positive since $\partial^{2} g(\theta, n) / \partial n \partial \theta \geq 0$ and $n^{T}>\bar{n}\left(I_{2}\right)$. The third and fourth terms are also positive given our assumptions on the functions $g(),. f($.$) and P($.$) ; finally, the last term is$ positive provided that the probability of correct screening increases with $\theta$ : $\left[\frac{\partial g\left(\theta, \bar{n}\left(I_{2}\right)\right)}{\partial \theta}+\frac{\partial g\left(\theta, \bar{n}\left(I_{2}\right)\right)}{\partial n} \frac{\partial \bar{n}\left(I_{2}\right)}{\partial P} \frac{d P}{d \theta}\right] \geq 0$. Moreover,

$$
\frac{\partial(\cdot)}{\partial n}=-\frac{\partial g(\theta, n)}{\partial n}(f(0)-f(1)) \Delta+P>0 .
$$

Thus it follows that if the probability of correct screening increases with $\theta$, then $d n^{T} / d \theta<0$.

Consider now the behavior of the liquidity-constrained true innovator. Since, $d P / d \theta \geq 0$ then $\bar{n}\left(I_{2}\right)$ is (weakly) decreasing in $\theta$. Hence, the statement of the proposition follows.

Let us now focus on the high-imitation-cost case. In this scenario, we can exploit the fact that the false type is indifferent between $\bar{n}\left(I_{2}\right)$ and $n=0$; hence, $n^{T}$ can be defined in terms of the value of $n$ that makes the false type indifferent between $n=0$ and $n$; namely

$$
f(0) \Delta+r+K-[g(\theta, n) f(0) \Delta+(1-g(\theta, n)) f(1) \Delta+r+K-n P]=0 .
$$


The rest of the proof is similar to the one we just presented for the lowcost-imitation case and it is therefore omitted for the sake of brevity. $\square$

\section{Proof of Proposition 3}

From Proposition 2 we know that the true types reduce the number of applications they file; hence, we just need to focus on the false type. In low-imitation-cost case, it follows immediately that when $d P / d \theta \geq 0$ also the number of patents applied for by the false innovator $\bar{n}\left(I_{2}\right)$ is (weakly) decreasing in $\theta$. Let us focus now on the high-imitation-cost case. In this scenario, the false type plays mixed strategies and applies for $\bar{n}\left(I_{2}\right)$ patents with probability $h\left(\bar{n}\left(I_{2}\right)\right)$ and chooses $n=0$ with complementary probability. Again, when $d P / d \theta \geq 0, \bar{n}\left(I_{2}\right)$ is (weakly) decreasing in $\theta$; hence we just need to show that $h\left(\bar{n}\left(I_{2}\right)\right)$ is decreasing in $\theta$. The probability $h\left(\bar{n}\left(I_{2}\right)\right)$ is the value of $h$ such that the strategies $\bar{n}\left(I_{2}\right)$ and $n=0$ yield the same payoff to the false type:

$$
\begin{aligned}
g\left(\theta, \bar{n}\left(I_{2}\right)\right) f(0) \Delta+\left(1-g\left(\theta, \bar{n}\left(I_{2}\right)\right)\right) f\left(\xi_{\bar{n}\left(I_{2}\right)}\right) \Delta+r+K-\bar{n}\left(I_{2}\right) P & =f(0) \Delta+r+K \\
\Leftrightarrow\left(1-g\left(\theta, \bar{n}\left(I_{2}\right)\right)\right)\left(f\left(\xi_{\bar{n}\left(I_{2}\right)}\right)-f(0)\right) \Delta+I_{2}-K & =0
\end{aligned}
$$

where $\xi_{\bar{n}\left(I_{2}\right)}=p_{2} /\left[p_{2}+p_{3}\left(1-g\left(\theta, \bar{n}\left(I_{2}\right)\right)\right) h\right]$, and where we have used the fact that $K-\bar{n}\left(I_{2}\right) P=I_{2}$.

Calling $(\cdot)$ the expression in (6), from the implicit function theorem it follows that $d h\left(\bar{n}\left(I_{2}\right) / d \theta=-(d(\cdot) / d \theta) /(d(\cdot) / d h)\right.$, where

$$
\frac{d(\cdot)}{d h}=\left(1-g\left(\theta, \bar{n}\left(I_{2}\right)\right)\right) \frac{d f\left(\xi_{\bar{n}\left(I_{2}\right)}\right)}{d \xi} \frac{d \xi_{\bar{n}\left(I_{2}\right)}}{d h}<0,
$$

since $\xi_{\bar{n}\left(I_{2}\right)}$ decreases with $h$. Moreover,

$$
\begin{aligned}
\frac{d(\cdot)}{d \theta}= & \left\{\frac{\partial g\left(\theta, \bar{n}\left(I_{2}\right)\right)}{\partial \theta}+\frac{\partial g\left(\theta, \bar{n}\left(I_{2}\right)\right.}{\partial n} \frac{\partial \bar{n}\left(I_{2}\right)}{\partial P} \frac{d P}{d \theta}\right\} \\
& {\left[\left(1-g\left(\theta, \bar{n}\left(I_{2}\right)\right)\right) \frac{d f}{d \xi_{\bar{n}\left(I_{2}\right)}} \frac{\partial \xi_{\bar{n}\left(I_{2}\right)}}{\partial g\left(\theta, \bar{n}\left(I_{2}\right)\right)} \frac{\partial g\left(\theta, \bar{n}\left(I_{2}\right)\right)}{\partial \theta}-\left(f\left(\xi_{\bar{n}\left(I_{2}\right)}\right)-f(0)\right)\right] \Delta . }
\end{aligned}
$$

When the probability of correct screening is increasing in $\theta$, it follows that $d h\left(\bar{n}\left(I_{2}\right) / d \theta \leq 0\right.$ provided that

$$
f\left(\xi_{\bar{n}\left(I_{2}\right)}\right)-f(0) \geq\left(1-g\left(\theta, \bar{n}\left(I_{2}\right)\right)\right) \frac{d f}{d \xi_{\bar{n}\left(I_{2}\right)}} \frac{\partial \xi_{\bar{n}\left(I_{2}\right)}}{\partial g\left(\theta, \bar{n}\left(I_{2}\right)\right)} \frac{\partial g\left(\theta, \bar{n}\left(I_{2}\right)\right)}{\partial \theta}
$$


Hence, the above condition is sufficient to guarantee that the expected number of applications filed by the false innovator $\left(h\left(\bar{n}\left(I_{2}\right)\right)\right.$ in the highimitation-cost case decreases as $\theta$ gets larger.

\section{Proof of Proposition 4}

We prove Proposition 4 by means of three claims. We focus on the case in which $\bar{n}_{g}\left(I_{2}\right) \equiv\left(K-I_{2}\right) / P_{g}$ and $\bar{n}_{r}\left(I_{2}\right) \equiv\left(K-I_{2}\right) / P_{r}$ are small enough so that the false type imitates the liquidity-constrained true innovator with probability 1 (i.e. we focus on the low-imitation-cost case). A similar reasoning applies to the case in which the false type plays mixed strategies (high-imitation-cost case).

Claim 1: Lemmas 1, 2 and 3 still apply.

Proof. One can easily check that Lemma 1 applies "within the tier", that is if $n_{i}$ is chosen with some positive probability by the false innovator and by at least one of the true types, then the true types prefer to apply for $n_{i}+\varepsilon$ patents rather than for $n_{i}$, with $i=r, g$. Lemmas 2 and 3 continue to hold since Assumption 2 is assumed to be satisfied for both gold-plate and regular patents.

Claim 2: the liquidity-constrained true innovator applies for either $\bar{n}_{r}\left(I_{2}\right)$ regular patents or for $\bar{n}_{g}\left(I_{2}\right)$ gold plate patents and the false type imitates it with probability 1.

Proof. Lemma 4 applies "within the tier" i.e. it shows that it cannot be that the liquidity-constrained true innovator applies for $n_{i}<\bar{n}_{i}\left(I_{2}\right)$ patents of type $i=r, g$. Hence, the liquidity-constrained true innovator applies for either $\bar{n}_{g}\left(I_{2}\right)$ gold-plate patents or for $\bar{n}_{r}\left(I_{2}\right)$ regular patents. Notice that the total expenditure in patenting is the same, $\bar{n}_{g}\left(I_{2}\right) P_{g}=\bar{n}_{r}\left(I_{2}\right) P_{r}=K-I_{2}$, hence, by Assumption 3, the two choices generate the same probability that the false type is detected $g\left(\theta_{g}, \bar{n}_{g}\left(I_{2}\right) P_{g}\right)=g\left(\theta_{r}, \bar{n}_{r}\left(I_{2}\right) P_{r}\right)$. This fact implies that there are two possible equilibria that are payoff equivalent: the liquidityconstrained true innovator and the false type apply $\bar{n}_{r}\left(I_{2}\right)$ regular patents or they both apply for $\bar{n}_{g}\left(I_{2}\right)$ gold-plate patents. Consider the first equilibrium for instance; both types choosing $\bar{n}_{r}\left(I_{2}\right)$ regular patents is supported by the following out of equilibrium belief: if $\bar{n}_{g}\left(I_{2}\right)$ is observed then, in case the PTO does not certify the firm to be the false innovator, $\xi_{\bar{n}_{g}\left(I_{2}\right)}=0$ which satisfies the D1 criterion. As a matter of fact, by definition of $\bar{n}_{g}\left(I_{2}\right)$ and $\bar{n}_{r}\left(I_{2}\right)$ the false and the liquidity-constrained types benefit from choosing $\bar{n}_{g}\left(I_{2}\right)$ whenever the associated out-of-equilibrium belief (in the case the PTO does not certify the firm to be the false type) is $\xi_{\bar{n}_{g}\left(I_{2}\right)}>\xi_{\bar{n}_{r}\left(I_{2}\right)}$; hence, the D1 criterion is silent in this case and, therefore, $\xi=0$ trivially satisfies it.

Claim 3: the deep-pocket true innovator applies for $n_{g}^{T}$ gold plate patents or $n_{r}^{T}$ for regular patents with $n_{g}^{T} P_{g}=n_{r}^{T} P_{r}$. 
Proof. Call $n_{r}^{T}$ the number of regular patents that makes the false type indifferent between the equilibrium strategy $\bar{n}_{r}\left(I_{2}\right)$ and imitating the deeppocket true innovator by filing $n_{r}^{T}$ patents; formally, $n_{r}^{T}$ is the value of $n_{r}$ such that:

$$
\begin{gathered}
g\left(\theta_{r}, \bar{n}_{r}\left(I_{2}\right) P_{r}\right) f(0) \Delta+\left(1-g\left(\theta_{r}, \bar{n}_{r}\left(I_{2}\right) P_{r}\right)\right) f\left(\xi_{\bar{n}_{r}\left(I_{2}\right)}\right) \Delta+r+K-\bar{n}_{r}\left(I_{2}\right) P_{r}= \\
g\left(\theta_{r}, n_{r} P_{r}\right) f(0) \Delta+\left(1-g\left(\theta_{r}, n_{r} P_{r}\right)\right) f(1) \Delta+r+K-n_{r} P_{r} .
\end{gathered}
$$

Similarly, call $n_{g}^{T}$ the number of gold-plate patents that makes the false type indifferent between the equilibrium strategy $\bar{n}_{r}\left(I_{2}\right)$ and imitating the deep-pocket true innovator by filing $n_{g}^{T}$ patents; formally, $n_{g}^{T}$ is the value of $n_{g}$ such that:

$$
\begin{gathered}
g\left(\theta_{r}, \bar{n}_{r}\left(I_{2}\right) P_{r}\right) f(0) \Delta+\left(1-g\left(\theta_{r}, \bar{n}_{r}\left(I_{2}\right) P_{r}\right)\right) f\left(\xi_{\bar{n}_{r}\left(I_{2}\right)}\right) \Delta+r+K-\bar{n}_{r}\left(I_{2}\right) P_{r}= \\
g\left(\theta_{g}, n_{g} P_{g}\right) f(0) \Delta+\left(1-g\left(\theta_{g}, n_{g} P_{g}\right)\right) f(1) \Delta+r+K-n_{g} P_{g} .
\end{gathered}
$$

Since by Assumption $3 g\left(\theta_{g}, x\right)=g\left(\theta_{r}, x\right)$ for all $x>0$, it follows that $n_{g}^{T} P_{g}=n_{r}^{T} P_{r}$; hence, $n_{g}^{T}$ and $n_{r}^{T}$ are payoff equivalent and the deep-pocket true innovator is indifferent between the two strategies.

\section{Proof of Proposition 5}

Consider the low-imitation-cost case and let $e^{*}$ be the optimal effort level, that is the value $e$ that satisfies the first order condition of the maximization problem stated in the text:

$$
p_{1}(f(1) \Delta+r) l^{\prime}(e)+p_{2}\left(f\left(\xi_{\bar{n}\left(I_{2}\right)}(\theta)\right) \Delta+r\right) l^{\prime}(e)-c^{\prime}(e)=0,
$$

where $\xi_{\bar{n}\left(I_{2}\right)}=p_{2} /\left[p_{2}+p_{3}\left(1-g\left(\theta, \bar{n}\left(I_{2}\right)\right)\right)\right]$. Denoting by $(\cdot)$ the expression in (6), from the implicit function theorem it follows that $d e^{*} / d \theta=$ $-(d(\cdot) / d \theta) /(d(\cdot) / d e)$, where

$$
\frac{d(\cdot)}{d \theta}=p_{2} \Delta l^{\prime}(e) \frac{d f\left(\xi_{\bar{n}\left(I_{2}\right)}\right)}{d \xi} \frac{d \xi_{\bar{n}\left(I_{2}\right)}}{d g}\left[\frac{\partial g\left(\theta, \bar{n}\left(I_{2}\right)\right)}{\partial \theta}+\frac{\partial g\left(\theta, \bar{n}\left(I_{2}\right)\right)}{\partial n} \frac{\partial \bar{n}\left(I_{2}\right)}{\partial P} \frac{d P}{d \theta}\right] .
$$

The above expression is positive provided that the probability of correct screening increases with $\theta:\left[\frac{\partial g\left(\theta, \bar{n}\left(I_{2}\right)\right)}{\partial \theta}+\frac{\partial g\left(\theta, \bar{n}\left(I_{2}\right)\right)}{\partial n} \frac{\partial \bar{n}\left(I_{2}\right)}{\partial P} \frac{d P}{d \theta}\right]>0$. In turn, the derivative $d(\cdot) / d e$ is negative by the concavity of the profit function. Hence, it follows that $d e^{*} / d \theta>0 . \square$

\section{Proof of Proposition 6}


Consider the false type; by choosing $\bar{n}\left(I_{2}\right)$ it obtains

$$
\begin{aligned}
& \int_{0}^{\bar{n}\left(I_{2}\right)}\left[\xi_{\bar{n}\left(I_{2}\right)}(m) \phi_{F}\left(m / \bar{n}\left(I_{2}\right)\right) R+\left(1-\xi_{\bar{n}\left(I_{2}\right)}(m)\right) \phi_{F}\left(m / \bar{n}\left(I_{2}\right)\right) r\right] d m-\bar{n}\left(I_{2}\right) P= \\
& \Delta \int_{0}^{\bar{n}\left(I_{2}\right)}\left[\frac{\bar{n}\left(I_{2}\right)}{3 \bar{n}\left(I_{2}\right)-2 m} \frac{2\left(\bar{n}\left(I_{2}\right)-m\right)}{\bar{n}\left(I_{2}\right)^{2}}\right] d m+r-\bar{n}\left(I_{2}\right) P= \\
& (1-(\ln 3) / 2) \Delta+r-\bar{n}\left(I_{2}\right) P .
\end{aligned}
$$

Given the beliefs specified in Proposition 1 the only sensible deviation for the false innovator is to choose $n=0$ with an associated payoff equal to $r$. A simple comparison of the payoffs reveals that choosing $\bar{n}\left(I_{2}\right)$ in accordance with the equilibrium is preferred provided that $\bar{n}\left(I_{2}\right) P<(1-(\ln 3) / 2) \Delta$. Moreover, $n^{T}$ is the value of $n$ that makes indifferent the false type between choosing the equilibrium strategy and mimicking the deep-pocket true innovator (with an associated payoff of $R-n^{T} P$ ). A simple computation reveals that

$$
n^{T}=\frac{(\ln 3)(R-r) / 2+\bar{n}\left(I_{2}\right) P}{P} .
$$

In turn, the liquidity-constrained true innovator chooses $\bar{n}\left(I_{2}\right)$ and obtains an expected payoff

$$
\begin{aligned}
& \int_{0}^{\bar{n}\left(I_{2}\right)}\left[\xi_{\bar{n}\left(I_{2}\right)}(m) \phi_{T}\left(m / \bar{n}\left(I_{2}\right)\right) R l+\left(1-\xi_{\bar{n}\left(I_{2}\right)}(m)\right) \phi_{T}\left(m / \bar{n}\left(I_{2}\right)\right) r l\right] d m-\bar{n}\left(I_{2}\right) P= \\
& \Delta l \int_{0}^{\bar{n}\left(I_{2}\right)}\left[\frac{\bar{n}\left(I_{2}\right)}{3 \bar{n}\left(I_{2}\right)-2 m} \frac{1}{\bar{n}\left(I_{2}\right)}\right] d m+r l-\bar{n}\left(I_{2}\right) P= \\
& (\ln 3) \Delta l / 2+r l-\bar{n}\left(I_{2}\right) P .
\end{aligned}
$$

Given the beliefs specified in Proposition 1 (and given that it is unable to apply for $n^{T}$ patents by assumption) the best possible deviation is $n=0$, with an associated payoff of $r l$. The equilibrium strategy $\bar{n}\left(I_{2}\right)$ is preferred to deviating and playing $n=0$ provided that $\bar{n}\left(I_{2}\right) P \leq(\ln 3) \Delta l / 2$, a condition which is verified when $\bar{n}\left(I_{2}\right) P<(1-(\ln 3) / 2) \Delta$.

Consider now the deep-pocket true innovator. By applying for $n^{T}$ patents as specified in the equilibrium it obtains $R l-n^{T} P=(\ln 3)(R-r) / 2-\bar{n}\left(I_{2}\right) P$. Given the beliefs specified in Proposition 1 the only sensible deviations for the deep-pocket true innovator is to choose either $n=0$ or $\bar{n}\left(I_{2}\right)$. The equilibrium strategy is preferred to $n=0$ (associated payoff of $r l$ ) provided 
that $\bar{n}\left(I_{2}\right) P<(l-(\ln 3) / 2) \Delta$, a condition which is verified when $\bar{n}\left(I_{2}\right) P<$ $(1-(\ln 3) / 2) \Delta$. By choosing $\bar{n}\left(I_{2}\right)$ the deep-pocket true innovator obtains (7); comparing the payoff associated with the strategies $n^{T}$ and $\bar{n}\left(I_{2}\right)$ one can check that the former is preferred by the deep-pocket true innovator whenever $l>(\ln 3) /(2-\ln 3)$. $\cdot$

\section{Appendix (2): microfoundations}

In this section, we present three possible microfoundations of the probabilities $f(\xi)$ and $1-f(\xi)$ defined in the model (Section 2).

\subsection{Entrant case}

Consider the setting described in Section 2 for the first two stages and assume that by investing $i$ the (incumbent) firm undertakes a process innovation that reduces its cost. If the firm innovates the (constant) marginal cost is $j$;

otherwise the marginal cost is $\bar{j}$, with $0 \leq j<\bar{j}$. The third stage is composed of two sub-stages as follows:

$t=3.1$ : entry decision. There is a potential entrant which decides whether or not to enter the market. Before taking its decision, the potential entrant observes the number of patents applied for by the incumbent, it observes the PTO's decision and forms its beliefs $\xi$ as specified in Section 2; moreover, the entrant observes the costs it needs to sink in order to enter the market, $\rho \chi$. The value of $\chi$ is common knowledge while the realization of $\rho$ is private information of the entrant and the incumbent only knows that $\rho$ is distributed according to a $U(0,1)$.

$t=3.2$ : competition. If the entrant did not enter the market, then: the incumbent is the monopolist and obtains profits $R l$ (in the case it has innovated and operates with the marginal cost $j$ ) or $R$ (in the case it has not innovated and operates with the cost $\bar{j}$ ) while the entrant obtains 0 . If instead the entrant entered the market, we assume that the marginal production cost of the incumbent $(j$ or $\bar{j}$ ) becomes common knowledge and that firms compete. The profits of the incumbent and the entrant are respectively $r l$ and $\pi_{E}(j)$ when the incumbent operates with the marginal cost $j$ and $r$ and $\pi_{E}(\bar{j})$ when the incumbent operates with the marginal cost $\bar{j}$. We assume that $\pi_{E}(\bar{j})>\pi_{E}(j)$ i.e. the profits of the entrant are larger when the incumbent operates with the high marginal cost $\bar{j}$. 
Consider the entry decision at $t=3.1$. The entrant enters the market provided that:

$$
\xi \pi_{E}(j)+(1-\xi) \pi_{E}(\bar{j})-\rho \chi \geq 0 \text { iff } \rho \leq \frac{\xi \pi_{E}(j)+(1-\xi) \pi_{E}(\bar{j})}{\chi} \equiv \rho(\xi) .
$$

Notice that, since $\pi_{E}(\bar{j})>\pi_{E}(j)$, then $\rho(\xi)$ decreases with $\xi$.

Consider now the decisions taken by the incumbent at $t=1$ and $t=2$. The incumbent knows that $\rho$ is uniformly distributed over $(0,1)$. Moreover, it anticipates that entry occurs with probability $\rho(\xi)$ and that this probability decreases with $\xi$ (we are implicitly assuming that $\rho(\xi) \in(0,1)$ ). The probability $\rho(\xi)$ corresponds to the probability $1-f(\xi)$ in Section 2 ; hence we derived a setting where $f(\xi)$ is differentiable and increasing in the beliefs.

\subsection{Venture capitalist}

Consider an alternative setting consistent with our model. At $t=3$, in order to bring the innovation to market, the firm needs some financial aid from a venture capitalist (VC). Conditional on being financed by the $\mathrm{VC}$, the true innovator receives a payoff $R l$ while the false innovator obtains $R$, with $l \geq 1$. If not financed, the firm (true or false innovator) obtains 0 (hence, $r l=r=0$ according to the notation of the model).

More specifically, we assume that at $t=3$ the firm is matched with a venture capitalist and it makes a take-it-or-leave-it proposal $s$ : the VC obtains a share $s \in[0,1]$ of the profits (the firm obtains the remaining $1-s$ share). The cost borne by the VC in order to finance the firm is $\rho$. The exact realization of $\rho$ is privately observed by the venture capitalist before deciding whether or not to accept the proposal of the firm; in turn, the firm knows that $\rho$ is uniformely distributed over the interval $(0,1)$.

The VC decides whether or not to accept the proposal after observing the following information: its type $\rho$, the number of patents applied for by the firm, the PTO's decision, the contract $s$ proposed by the firm. Denoting by $\xi \in[0,1]$ the belief that it holds when taking its decision, then the venture capitalist accepts the proposal of the firm provided that:

$$
\xi s R l+(1-\xi) s R-\rho \geq 0 \text { iff } \rho \leq \xi s R l+(1-\xi) s R \equiv \rho(\xi, s),
$$

where $\rho(\xi, s)$ is increasing in $\xi$ and in $s$. 
When offering contract $s$ the firm anticipates that its proposal is going to be accepted with probability $\rho(\xi, s)$ (we are implicitly assuming that $\rho(\xi, s) \in$ $(0,1))$.

Consider the firm that makes the proposal $s$ knowing that the VC holds the belief $\xi$. The firm anticipates that the proposal is going to be accepted with probability $\rho(\xi, s)$ and its expected profits are $\rho(\xi, s)(1-s) R l$ (in the case of true innovator) and $\rho(\xi, s)(1-s) R$ (in the case of false innovator). Moreover, observe that at $t=3$ we do not need to distinguish between the liquidity-constrained and the deep-pocket true innovators given that they have the same strategy set and they expect the same payoff (net of $i$ and of the patenting fees). Hence, at stage $t=3$ there are only two firm types, the true and the false innovator.

\subsubsection{Equilibrium of the sub-game at $t=3$}

At $t=3$, given that the market holds the belief $\xi$, the true innovator expects to obtain $\rho(\xi, s)(1-s) R l$ : it receives the financial aid with probability $\rho(\xi, s)$ and it obtains a share $(1-s)$ of the expected profits $R l$. Similarly the false innovator expects to obtain $\rho(\xi, s)(1-s) R$. Hence the two types choose $s$ in order to maximize $\rho(\xi, s)(1-s)$. The following is the equilibrium of the subgame played at $t=3$ :

i) the two types of the firm propose $s^{\xi}=\arg \max _{s} \rho(\xi, s)(1-s)$ and the VC maintains the belief $\xi$ formed at $t=2$;

ii) the $\mathrm{VC}$ holds the belief $\xi=0$ when receiving a proposal $s \neq s^{\xi}$.

Proof. Given that $\rho(\xi, s)$ increases with the belief $\xi$ and given the out-of-equilibrium beliefs of the $\mathrm{VC}$, both types of the firm prefer $s^{\xi}$ to any possible deviation. Moreover, notice that the D1 criterion is silent about the out-of-equilibrium beliefs, and therefore the beliefs specified in part ii) satisfy it; one can easily check this last point since both types benefit from proposing $s^{\prime} \neq s^{\xi}$ if and only if the associated belief $\xi^{\prime}$ is such that $\rho\left(\xi^{\prime}, s^{\prime}\right)\left(1-s^{\prime}\right)>\rho\left(\xi, s^{\xi}\right)\left(1-s^{\xi}\right)$. Hence the two types of the firm would deviate under exactly the same circumstances.

The function $f(\xi)$ defined in Section 2 of the model corresponds to $\rho\left(\xi, s^{\xi}\right)\left(1-s^{\xi}\right)$ where $s^{\xi}$ is the equilibrium proposal defined above. Simple calculations show that $s^{\xi}=1 / 2$ and that $\rho\left(\xi, s^{\xi}\right)\left(1-s^{\xi}\right)=(1 / 4)(\xi R l+(1-\xi) R)$; this latter function is differentiable and increasing in $\xi$. Hence we have derived a setting where $f(\xi)$ is differentiable and increasing in $\xi$. 


\subsection{Licensing}

A setting similar to the case of venture capital is the following one. Suppose that the firm is unable to exploit its innovation commercially and therefore, at time $t=3$, once the previous decisions have been taken, it sells/licenses the innovation to a licensee. More specifically, we assume the firm is matched with a licensee, and then it proposes the following royalty contract: the licensee purchases the innovation and pays the firm a share $(1-s)$ of the profits generated by the innovation (and keeps the remaining share $s$ ). We assume that licensees differ in terms of the ability to commercially exploit the innovation: the overall profits generated by the innovation equal $\rho R l$ (in case of true innovator) or $\rho R$ (in case of false innovator), with $l \geq 1$. The exact realization of $\rho$ is private information of the licensee while it is publicly known that $\rho$ is distributed according to a $U(0,1)$. If the innovation is not licensed, then the firm obtains no profit (hence, $r l=r=0$ in terms of the notation of the model).

The licensee decides whether or not to accept the royalty contract $s$ after observing the following information: its type $\rho$, the number of patents applied for by the firm, the PTO's decision, the contract $s$ proposed by the firm. Denoting by $\xi \in[0,1]$ the belief that it holds when taking its decision, then the licensee accepts the proposal of the firm provided that:

$$
\xi s \rho R l+(1-\xi) s \rho R \geq \underline{U} \text { iff } \rho \geq \frac{\underline{U}}{\xi s R l+(1-\xi) s R} \equiv \rho(\xi, s),
$$

where $\rho(\xi, s)$ is decreasing in $\xi$ and in $s$ and where $\underline{U}$ is the reservation utility of the licensee.

When offering the contract $s$ the firm anticipates that its proposal is going to be accepted with probability $1-\rho(\xi, s)$ (we are implicitly assuming that $\rho(\xi, s) \in(0,1))$.

By applying the same arguments used in the case of venture capital, it would be easy to show that one can derive $f(\xi)$ which is differentiable and increasing in the beliefs; for the sake of brevity we omit this part. 


\section{References}

Anton, J.J. and Yao D. A. (2003). Patents, Invalidity, and the Strategic Transmission of Enabling Information." Journal of Economics and Management Strategy, 23(2):151-178.

Anton, J.J. and Yao D. A. (2004). Little patents Big Secrets: Managing Intellectual Property. RAND Journal of Economics, 35(1):1-22.

Atal, V. and Bar, T. (2014). Patent quality and a Two-tiered patent System. The Journal of Industrial Economics, 62(3):503-540.

Banks, J. and Sobel, J. (1987). Equilibrium Selection in Signaling Games. Econometrica, 55(3):647-661.

Caillaud, B. and Duchene, A. (2011). Patent Office in innovation policy: Nobody's perfect. International Journal of Industrial Organization, 29(2):242-252.

Cho, I. and D. Kreps (1987) Signaling games and stable equilibrium. Quarterly Journal of Economics, 102(2):179-222.

Choi, J. (2005). Live and let live: a tale of weak patents. Journal of the European Economics Association, 3 (2-3):724-733.

Cockburn, I. and MacGarview, M. (2009). Patents, Thickets and the Financing of Early-Stage Firms: Evidence from the Software Industry. Journal of Economics and Management Strategy, 18:729-773.

Conti, A., Thursby, J. and Thursby, M. C. (2013a). Patents as signal for startup financing. Journal of Industrial Economics, 61(3):592-622.

Conti, A., Thursby, J. and Rothaermel, F.T. (2013b). Show me the right stuff: Signals fro high-tech startups. Journal of Economics and Management Strategy, 22(2):41-364.

Encaoua, D., Guellec, D. and Martinez, C. (2006). Patent systems for encouraging innovation: Lessons from economic analysis. Research Policy, 35(9):1423-1440.

Fabrizi, S., Lippert, S. Norback, P.H. and L. Persson (2013). Venture capitalists and the patenting of innovations. Journal of Industrial Economics, 61(3):623-659.

Farrel, J. and Shapiro C. 2008). How Strong are Weak Patents? American Economic Review, 98(4):1347-1369.

Gambardella, A. (2013) The Economic Value of Patented Inventions: Thoughts and some open questions. International Journal of Industrial Organization, 31(2):626-633.

Graham, S.J., Merges, R.P., Samuelson, P., and Sichelman, T. (2010). High Technology entrepreneurs and the patent system: Results of the 2008 Berkeley Patent Survey. Berkeley Technology Law Journal, 24(4):1255-1328.

Greenberg, G. (2013). Small Firms, Big Patents? Estimating patent 
Value using Data on Israeli Start-up Firms' Financing Rounds, European Management Review, 10(4):183-196.

Hall, B. H. and Ziedonis R. H. (2001) The patent paradox revisited: an empirical study of patenting in the U.S. semiconductor industry, 1979-1995. RAND Journal of Economics, 32(1):101-128.

Hall, B. H, Helmers, C., Rogers, M. and Sena V. (2013). The Importance (or not) of patents to UK firms. NBER Working Paper 19089.

Häussler, C., Harhoff, D. and Müeller E. (2009). To be financed or not...The role of patents for venture capital financing. CEPR Discussion Paper Series 7115.

Harhoff, D. and S. Wagner (2009). The duration of patent examination at the European Patent Office. Management Science, 55(12):1969-1984.

Hemphill, C.S. and Sampat, B.N. (2012). Evergreening, patent challenges, and effective market life in pharmaceuticals. Journal of Health Economics, 31(2):327-339.

Hsu, D. and Ziedonis, R. (2008). Patents as quality signals for entrepreneurial ventures. Academy of Management Proceedings. Vol. 2008. No. 1.

Jansen, J. (2011) On competition and the strategic management of intellectual property in oligopoly. Journal of Economics and Management Strategy, 20(4):1043-1072.

Koenen, J. and Peitz, M. (2013). Firm Reputation and Incentives to "Milk" Pending Patents. CESifo Working Papers 4355.

Kou, Z., Rey, P. and Wang T. (2013). Non-Obviousness and Screening. The Journal of Industrial Economics, 61(3):700-732.

Lemley, M. Lichtmand, D., and Sampat, B. (2005). What to do about bad patents? Regulation, Winter:10-13.

Lemley, M. and Sampat, B. (2008). Is the patent office a rubber stamp? Emory Law Journal, 58:181-202.

Long, C. (2002). Patent signals. University of Chicago Law Review, (69):625-679.

Mann, R.J. (2005). Do patents facilitate financing in the software industry? Texas Law Review, 83(4):961-1030.

O’Donoghue, T. (1998). A patentability requirement for sequential innovation. RAND of Economics, 29(4):654-679.

O'Donoghue, T., Scotchmer, S., and Thisse, J. (1998). Patent breadth, patent life, and the pace of technological improvement. Journal of Economics and Management Strategy, 7:1-32.

Ouellette, L.L. (2010). How many patents does it take to make a drugfollow-on pharmaceutical patents and university licensing. Mich. Telecomm. छTech. Law Review, 17:299-336. 
Roland Berger (2005). A Study on the Cost of Patenting, prepared on behalf of the European Patent Office, Munich: Roland Berger Market Research.

Schankerman, M. (2013). Introduction. Journal of Industrial Economics, 61(3):471-480.

Schuett, F. (2013). Inventors and Impostors: An Analysis of Patent Examination with Self-Selection of Firms into R\&D." Journal of Industrial Economics, 61(3):660-699.

Thomas, J. R. (2001). Collusion and Collective Action in the Patent System: A Proposal for Patent Bounties. University of Illinois Law Review, 2001(1):305-353. 